\title{
Article \\ Designing a Sustainable Green Closed-Loop Supply Chain under Uncertainty and Various Capacity Levels
}

\author{
Mohsen Tehrani and Surendra M. Gupta *D \\ Department of Mechanical and Industrial Engineering, Northeastern University, Boston, MA 02115, USA; \\ Tehrani.m@northeastern.edu \\ * Correspondence: s.gupta@northeastern.edu
}

Citation: Tehrani, M.; Gupta, S.M. Designing a Sustainable Green Closed-Loop Supply Chain under Uncertainty and Various Capacity Levels. Logistics 2021, 5, 20. https:// doi.org/10.3390/logistics5020020

Academic Editor: Robert Handfield

Received: 17 February 2021

Accepted: 16 March 2021

Published: 7 April 2021

Publisher's Note: MDPI stays neutral with regard to jurisdictional claims in published maps and institutional affiliations.

Copyright: (c) 2021 by the authors. Licensee MDPI, Basel, Switzerland. This article is an open access article distributed under the terms and conditions of the Creative Commons Attribution (CC BY) license (https:// creativecommons.org/licenses/by/ $4.0 /)$.

\begin{abstract}
The ever-increasing concerns of the growth in the volume of waste tires and new strict government legislations to reduce the environmental impact of the end-of-life (EOL) tires have increased interest among companies to design a sustainable and efficient closed-loop supply-chain (CLSC) network. In the real world, the CLSC network design is subject to a variety of uncertainties, such as random and fuzzy (epistemic) uncertainties. Designing a reliable and environmentally cautious CLSC with consideration of risks and the uncertainty of the parameters in the network is necessary for a successful supply-chain network. This study proposes a sustainable and environmentally cautious closed-loop supply-chain network for the tire industry, by considering several recovery options, including retreading, recycling, and energy recovery. This study aims to design and develop a robust multi-objective, multi-product, multi-echelon, multi-cycle, multi-capacity, green closed-loop supply-chain network under hybrid uncertainty. There are two types of uncertainties associated with the parameters in the network. There is an uncertainty associated with the demand, which is expressed in some future scenarios according to the probability of their occurrences, as well as fuzzy-based uncertainty associated with return rates, retreading rates, recycling rates, procurement, and production costs, which are expressed with possibilistic distributions. In order to deal with this hybrid uncertainty, a robust fuzzy stochastic programming approach has been proposed, and the proposed mixed integer programming model is applied to a case study in the tire industry to validate the model. The result indicates the applicability of the proposed model and its efficiency to control the hybrid uncertainties and the risk level in the network.
\end{abstract}

Keywords: closed-loop supply chain; location-allocation problem; robust optimization; tire industry; multi-objective decision making; hybrid uncertainty; augmented epsilon constraint

\section{Introduction}

In today's business world, companies need to pay close attention to their supply-chain management systems and logistics network, in order to effectively compete in a highly competitive market. A great supply-chain system is a necessity for any manufacturing organization. Most manufacturers put their focus primarily on the forward supply chain; however, the key to success is to have a holistic view of the entire forward and reverse logistic network. Reverse logistics is a network where selected products transfer up the stream from the end users for repair, remanufacturing, or recycling [1]. Environmental concerns, government regulations, and the potential economic benefits are some of the motives for manufacturers to move toward this direction [2,3]. Besides environmental regulations, consumer's awareness and their responsibility against the environment are some of the motives. The tire industry is one of the industry sectors moving toward this direction. Worldwide, more than a billion tires are manufactured every year, and a significant number of them are thrown away after being used. According to the US Environmental Protection Agency (EPA), in the United States alone, 280 million tires are being scraped every year. The average passenger tire weighs about 22 pounds, and the 
average commercial semi-truck tire weighs about 110 pounds, and these tires contain oil and other combustible carbon compounds, which create a fire hazard. Figure 1. Tire fires are difficult to extinguish and can break the tire down into hazardous compounds, including gases, heavy metals, and oil. Tire piles can also create a breeding ground for pests such as rodents and mosquitos that contribute to the spread of diseases [4,5]. Although the forward tire supply chain is well developed, there is no systemic and comprehensive reverse logistics system in place. Most collection, retreading, and recycling centers are small individual shops with a limited capacity. Government regulations and requirements vary from state to state. This lack of a comprehensive plan can be seen by a large volume of tires still ending up in the landfills.
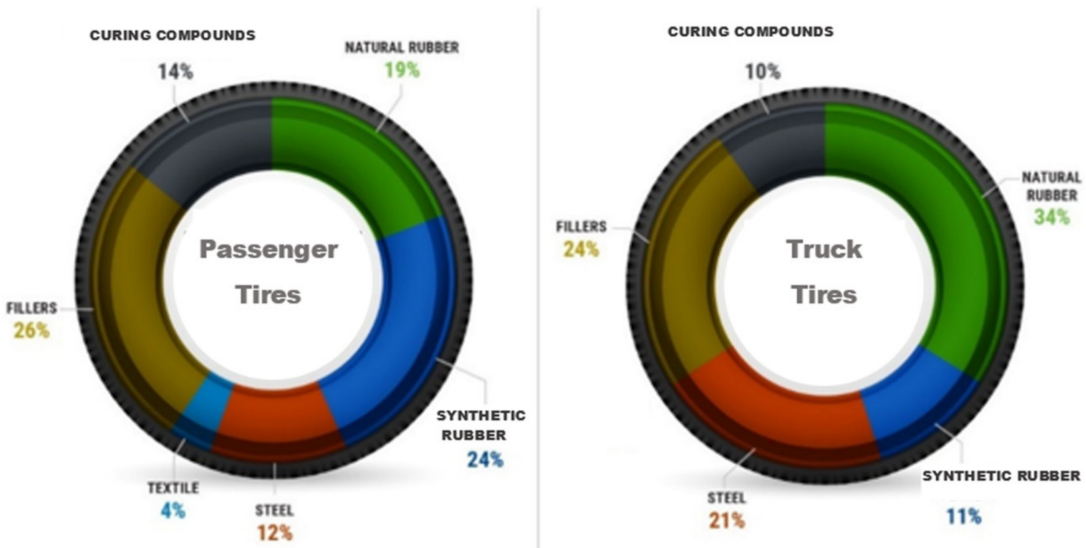

Figure 1. Tire composition.

There are many factors that are considered by the decision maker (DM) when designing a closed-loop supply-chain (CLSC) network, such as costs, environmental impacts, social dimensions, uncertainty in the parameters, governmental regulations, disruption and operational risks, reliability of the network, etc. Uncertainty with the parameters is probably the biggest challenge facing the supply-chain network (SCN) design. Random and fuzzy uncertainties are generally the two types of uncertainty in the design of a supplychain network [6]. Possibilistic, stochastic, and robust programming are typically the methods utilized to control these uncertainties. Stochastic programming can be used when there are sufficient data available to estimate the probability distribution of the uncertain parameters that is used for modeling the uncertain parameters under different scenarios in the network [7]. For instance, several scenarios for demand may be related to economic conditions, occurrence of natural disasters, changes in customer preferences, development of new technologies, etc. However, the value of specific parameters in each scenario could be imprecise and can be determined by possibilistic distribution.

Possibilistic programming is used when there are no sufficient historical data available, but the possibility distribution of uncertain parameters can be estimated by using the expert's knowledge in the field. Robust programming is a method that allows the DMs to adjust the risk level of output results with respect to the uncertainty of parameters [8]. Combining the stochastic, possibilistic, and robust programming can supplement the weaknesses of each individual method and provide more reliable results for the DMs in the supply-chain network.

In this study, robust fuzzy stochastic programming was used to cope with hybrid uncertainties in the model. Moreover, in this paper, we provide a holistic and realistic modeling approach for the coordination of a forward and reverse tire management system that extends the life span of re-manufacturable tires, recovers the value of scrap tires, and eliminates the disposal in the landfills. In that respect, we examine different recovery options simultaneously, such as retreading, recycling, and energy recovery. Our model aims to maximize the total CLSC profit, minimize the total greenhouse gas (GHG) generated along the CLSC and maximize the social responsibilities which is presented in the form of 
the total number of jobs created by expanding the network in the forward direction and establishing a new reverse logistics network.

As the body of literature about CLSC network design shows, most models ignore the fact that products can be remanufactured multiple times and remain in the supply-chain network to satisfy the customer demand, rather than being recycled prematurely. We refer to this as a multi-cycle supply-chain network. This can impact model assumptions and outputs with respect to overall market demand, the production rate of manufacturing centers, the return rate of end-of-life (EOL) products, and the capacity of the facilities in the network. Also, a genuinely green supply-chain network must consider and plan for the return of EOL remanufactured products for final processing. This has also been neglected in most previous studies.

This study aims to design a closed-loop supply-chain network considering the economic, environmental, and social factors with the following contributions:

- Proposing a new sustainable, robust, multi-objective, multi-product, multi-echelon, multi-cycle, multi-capacity, green closed-loop supply-chain network by considering the real-world situation where used tires are retreaded multiple times, and the retread tires are being returned to the collection center for final processing.

- Presenting a novel Robust Fuzzy/Possibilistic Stochastics Programming (RFSP) model that controls scenario-based uncertainty (disruption risks) and fuzzy-based uncertainty (operational risks) in the network simultaneously and provide a more reliable output results for the managers and the DMs.

- Evaluating the possibility and feasibility of expanding the existing forward logistic network at different capacity levels to cope with market demand under various scenarios, and also the feasibility of establishing a new reverse logistic network at different capacity levels to cope with the return rate of EOL tires.

- Validation of the proposed model by sensitivity analysis through a case study.

The paper is structured as follows. In Section 2, the relevant literature on closed-loop supply-chains network design and tire industry is reviewed; some works concerning uncertainty in the supply chain are also analyzed. In Section 3, the main problem characteristics, assumptions, and mathematical formulation are presented. In Section 4, a proposed Robust Fuzzy/Possibilistic Stochastics Programming (RFSP) approach and augmented epsilon constraint method are described in detail. In Section 5, the proposed model is applied to an example problem inspired by a case in the U.S. The computational results and the sensitivity analysis are also presented in this section. Finally, in Section 6, some conclusions and future work are discussed.

\section{Literature Review}

Designing a robust and flexible supply-chain network (SCN) that includes optimizing the number, capacity, and location of network facilities has received a lot of attention these days [9]. A closed-loop supply-chain network (CLSC) considers both forward and reverse supply-chain networks in an integrated manner. There have been many papers published in this subject area and especially green closed-loop supply-chain network. Govindan et al. (2014) and Devika et al. (2015) provided a comprehensive survey of the CLSC network $[10,11]$. Moreover, a comprehensive review on environmentally conscious manufacturing and product recovery was provided by Ilgin and Gupta (2010) [9]. Jindal and Sangwan (2016) presented the design and optimization of a multi-objective closed-loop supply chain, considering the economic and environmental factors under uncertainty using the interactive $\varepsilon$-constraint method [12]. Sarkar et al. (2017) studied the effect of transportation cost and carbon emission cost on remanufacturing. They proposed a multi-echelon closed-loop supply-chain model with third-party logistics (3PL) [13]. In another study, they developed a multi-attribute closed-loop supply-chain model for selfhealing polymers-based returnable transport packaging. Their proposed multi-objective supply-chain model aimed to maximize profit while minimizing carbon emission of the system [14]. 
Dulman and Gupta (2018) studied the financial impact of sensors on closed-loop supply-chain systems [15]. Zhou and Gupta (2019) provided a pricing strategy for new and remanufactured high-tech products. Their model aimed to maximize the profit associated with customer demand based on the manufacturer, retailer, and joint supply chain [16]. In another study, they proposed partial least square method to explore the factors that affect value depreciation rate and price differentiation between new and remanufactured iPhone and iPad [17]. Aldoukhi and Gupta (2019) proposed a new model for designing a closed-loop supply-chain network by considering downward product substitution policy under four carbon-emission regulation policies. They used robust optimization to deal with uncertainty related to product demand and number of returned products [18]. In another study, they proposed a multi-objective model to design a closed-loop supply-chain (CLSC) network, with the aim of minimizing the total cost, minimizing the carbon emission, and maximizing the service level of the retailers. They used physical programing approach to model the problem [19]. Fadhel and Gupta (2019) evaluated the food waste valorization alternatives from a sustainability point of view. They estimated energy utilization and GHG emission reduction for each potential food waste processing technique [20]. Gupta et al. (2019) studied the optimal control of production and maintenance operations in smart custom manufacturing systems, using a heuristic algorithm [21]. De and Giri (2020) studied a closed-loop supply chain (CLSC), focusing on managing, scheduling, and routing problems, to achieve economic and environmental sustainability [22]. Mogale et al. (2018) designed a dual market facility network by considering manufacturing the common components centrally and locally [23]. Oliveira and Machado (2021) provided a literature review on the application of optimization methods in a closed-loop supply chain [24]. Zahedi et al. (2021) proposed a closed-loop supply-chain network by considering multi-task sales agencies and multi-mode transportation, and they solved their model by using metaheuristic and hybrid algorithm [25].

Guarnieri et al. (2020) proposed a theoretical model to assess reverse logistics using multi-criteria decision-making for e-waste [26]. Vieira et al. (2020) studied the barriers in implementing reverse logistics for e-waste and investigated the widely applied multicriteria decision aid (MCDA) methods used to prioritize these barriers [27].

There have been many researches in the area of sustainability [28]. Mogale et al. (2019) designed a sustainable food-grain supply-chain distribution system with the aim of minimizing the cost and carbon dioxide emission simultaneously [29]. Ullah et al. (2020) investigated the optimal remanufacturing strategy and reusable packaging capacity under stochastic demand and return rate [30]. Ghadge et al. (2020) studied obstacles in sustainability implementation in cold-food supply-chain networks by considering a case of UK artisan cheese producers [31]. Gupta et al. (2020), presented a compilation of six recent papers on a variety of topics to demonstrate the pioneer research activity within responsible and sustainable manufacturing [32]. Sazvar et al. (2021) designed a sustainable CLSC for pharmaceutical by considering manufacturer's brand and waste management [33].

Inconsistent, unreliable, and uncertain data are one of the biggest challenges facing the SCN [34-36]. In the real world, there are many parameters in SCN that are uncertain, such as customer demands, costs of opening new facilities at a specific location, manufacturing cost, cost of raw material, transportation cost, etc. [37]. According to Mula et al. (2006/2007), the two main types of uncertainty are randomness and epistemic [38,39]. Stochastic programming and possibilistic programming are the most common methods to deal with randomness and epistemic (fuzzy) uncertainty, respectively [40,41]. A flexible robust optimization approach for scenario-based stochastic programming models was proposed by Mulvey et al. (1995) [42]. Their approach was later developed by $\mathrm{Yu}$ and Li, 2000 [7]. Pishvaee et al. (2018) also developed a new approach, called robust possibilistic programming [43]. Mousazadeh et al. used the robust possibilistic approach for a healthservice network design [44]. Tozanli and Gupta et al. (2017) provided a literature review paper for environmentally concerned logistics operation (ECLO) in fuzzy environment. They utilized over 800 papers between 1994 and 2017 and performed a detailed analysis 
of ECLO with an emphasis on fuzzy application [45]. Vahdat et al. (2017) provided a two-stage stochastic programming modeling to design a multi-period, multistage, and single-commodity CLSC network under uncertainty [46]. Parakash et al. (2018) proposed a CLSC network under risks and demand uncertainty [47]. Yolmeh and Saif (2020) provided a supply-chain network design with assembly and disassembly line balancing under uncertainty [48]. Wang et al. (2021) studied various incentive mechanism in green supply chain under demand uncertainty [49]. Tordecilla et al. (2021) provided a review of simulation optimization methods for designing a resilient supply chain under uncertainty [50].

There has been some research on the tire CLSC network. In 1997, Ferrer published a paper and estimated the optimal number of times each tire can be retreaded [51]. Sasikumar et al. (2010) provided an optimization model for a tire remanufacturing case [52]. According to Chopra and Meindl (2015), between 2009 and 2011, only 3 percent of total sales by US firms within the tire industry were retread tires [53]. One of the challenges facing the tire industry is the coordination of the entire forward and reverse logistics network and managing the flow of the new and used tires [54]. Amin et al. (2017) examined a case study for CLSC network for tire industry in Toronto, Canada. They developed a mixed integer linear programming model, under uncertainty, with a goal of maximizing the profit [55]. Subulan et al. (2015) examined tire remanufacturing case study in Turkey using a fuzzy mixed integer programming approach [56]. Derakhshan et al. (2017) presented a technique for recycling the tire waste, as well as reuse of the waste [57]. O'Brien and North (2017) investigated the emission of pollutants from tire waste. They studied the types of gases that are produced from car tire waste [58]. Fathollahi Fard et al. (2018) proposed a tri-level programming model for tire CLSC to identify the decision variable, using real datasets [59]. Pedram et al. (2018) used stochastic programing to design a single objective tire CLSC network [60]. Lokesh et al. (2018a) studied a tire-retreading supply-chain network under carbon tax policy. In another study, Lokesh et al. (2018b) used a fuzzy goal programming approach for Brownfield tire retreading case study under carbon tax policy [61,62]. SahebJamnia et al. (2018) also modeled a robust closed-loop supply chain of the tire industry and used hybrid metaheuristic algorithms to solve the model [63]. Abdolazimi et al. (2019) proposed a multi-objective closed-loop supply chain by integrating on time delivery and the cost [64]. Mehrjerdi at al. (2020) studied sustainability and resiliency of a closed-loop supply chain, using multiple sourcing strategy [65]. Lokesh et al. (2020) also designed a sustainable tire supply chain with a fuzzy goal planning approach [66].

\section{Tire Industry and Recovery Options}

According to Modern Tire Dealer (MTD), in 2019, 267.8 million new passenger tires, 37.9 new light truck tires, and 25.4 million new medium and heavy truck tires were shipped within the United States. EOL tires due to the large quantities being manufactured and the durability of the material being used, pose a significant challenge for the government and the supply chain. Disposing scrap tires in landfills consumes valuable space and can also result in the penetration of pollutants and metal contents into the underground water. With recent research and advancement in the composition of tire, tires have become even more durable. When the tire treads are worn out, the majority of the time, the tire casing is still in good condition and can be reused many times. Disposing of in the landfill or incineration for energy recovery only shortens the life span of the tire casing. There are few recovery options available for EOL tires, some of which are as follows: 
Reuse or repurposing the tire is the most environmentally friendly option that extends the life cycle of the tires. Tires with sufficient thread depth can be sold in the used tire market. Whole tires can also be used for a number of applications, including erosion control, playground equipment, crash barriers on the side of the highway, and boat bumpers at the docks.

Retread or recap is a process in which the worn thread of the tire is removed from the tire casing and replaced with new treads. Study shows 25 gallons of oil needed to produce a new truck tire compared to only 7 gallons of oil to retread a used one. That results in a significant reduction in greenhouse gas emission, preserving raw material, and minimizing the amount of waste disposed of in the landfills.

Waste tires are typically shredded before they are recycled. Recycled tires can be used as crumb rubber in asphalt pavement or as an aggregate in Portland cement concrete. Tires are also cut up and used in garden beds to hold in the water and to prevent weeds from growing [67].

A large percentage of scrap tires are used as fuel. Tire-derived fuel (TDF) is a fuel derived from scrap tires. Scrap tires, either shredded or as a whole, is used to supplement coal in power plants, cement kilns, paper mills, etc. Research has shown that atmospheric contamination dramatically increases when tire rubber is used as fuel [68-72].

Disposal in the landfill is the most harmful option due to the large volume and high percentage of void space inside the tire. Tires can trap gas that causes them to become buoyant and damage the landfill covers.

Designing an economically optimized and environmentally friendly CLSC network is a prerequisite for tire manufacturers not only to earn a profit but also to decrease waste, preserve natural resources, and save landfills and the environment, with the ultimate goal of sustainable development. This has motivated this study to take a closer look at the challenges facing the tire industry and come up with a solution that creates a win-win situation for companies in the tire industry network and the environment. Based on the reviewed papers reported in Table 1, in this study, we aimed to identify the uncertain parameters in the logistics network and find the solution for dealing with these uncertainties in order to reach a robust modeling in the logistics network. We introduce a robust multi-objective, multi-product, multi-echelon, multi-cycle, multi-capacity, green closed-loop supply-chain network under hybrid uncertainty with the aim of reducing costs, reducing environmental impacts, and increasing the social responsibilities and the reliability of the network. 
Table 1. Research comparison.

\begin{tabular}{|c|c|c|c|c|c|c|c|c|}
\hline & \multicolumn{3}{|c|}{ Network Type } & \multirow{2}{*}{$\begin{array}{l}\text { Supplier } \\
\text { Selection }\end{array}$} & \multirow{2}{*}{$\begin{array}{l}\text { Multi Raw } \\
\text { Material }\end{array}$} & \multirow{2}{*}{$\begin{array}{c}\text { Multi } \\
\text { Product }\end{array}$} & \multirow{2}{*}{$\begin{array}{c}\text { Multi Capacity } \\
\text { Level }\end{array}$} & \multirow{2}{*}{$\begin{array}{c}\text { Network } \\
\text { Expansion }\end{array}$} \\
\hline & Forward & Reverse & CLSC & & & & & \\
\hline Sasikumar et al. (2010) [52] & & $\mathrm{x}$ & & & & & & \\
\hline Pishvaee et al. (2012) [43] & $x$ & & & & & & & \\
\hline Subulan et al. (2015) [56] & & & $x$ & $x$ & $x$ & $x$ & $x$ & \\
\hline Jindal et al. (2016) [12] & & & $x$ & $x$ & & & & \\
\hline Amin et al. (2017) [55] & & & $x$ & $x$ & & $x$ & & \\
\hline Pedram et al. (2018) [60] & & & $x$ & & & $x$ & & \\
\hline Fazli-Khalaf et al. (2018) [36] & & & $x$ & $\mathrm{x}$ & $\mathrm{x}$ & & $\mathrm{x}$ & \\
\hline Fathollahi-Fard et al. (2018) [59] & & & $x$ & $x$ & & $x$ & & \\
\hline Sabouhi et al. (2018) [37] & $x$ & & & $x$ & $x$ & $\mathrm{x}$ & $x$ & \\
\hline Lokesh et al. (2018a) [61] & & $x$ & & & & $x$ & $x$ & \\
\hline Lokesh et al. (2018b) [62] & & $x$ & & & & $x$ & & \\
\hline Sahebjamnia et al. (2018) [63] & & & $\mathrm{x}$ & $\mathrm{x}$ & & $\mathrm{x}$ & & \\
\hline Yadollahinia et al. (2018) [54] & & & $x$ & & & $\mathrm{x}$ & & \\
\hline Ahmadi and Amin (2019) [35] & & & $x$ & $\mathrm{x}$ & $x$ & $\mathrm{x}$ & & \\
\hline Mogale et al. (2019) [29] & $x$ & & & & & & & \\
\hline De et al. (2020) [22] & & & $x$ & & & $x$ & & \\
\hline Lokesh et al. (2020) [66] & & $x$ & & $\mathrm{x}$ & $\mathrm{x}$ & $x$ & & \\
\hline This Paper & & & $x$ & $x$ & $x$ & $\mathrm{x}$ & $\mathrm{x}$ & $x$ \\
\hline
\end{tabular}


Table 1. Cont

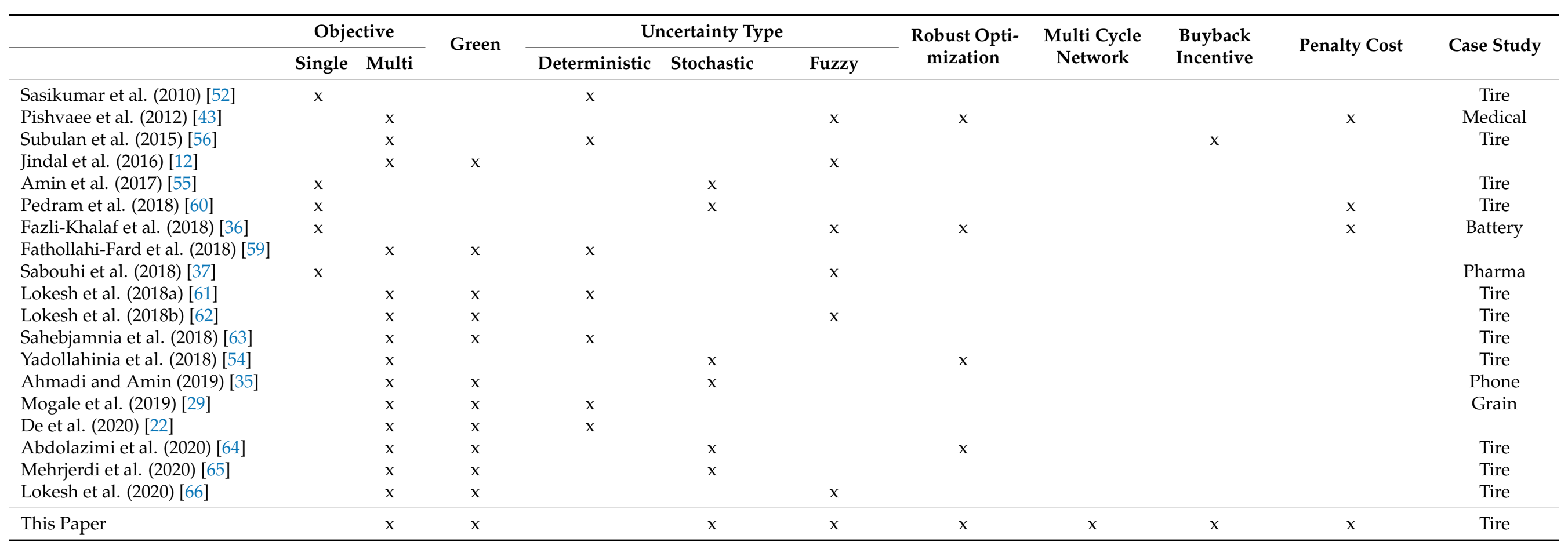




\section{Problem Description and Formulation}

As shown in Figure 2, we are considering a multi-product and multi-echelon CLSC network consisting of suppliers, production centers, distribution centers, collection centers, retreading centers, recycling, and energy-recovery centers. In the forward flow, various raw materials are procured from the available choice of suppliers. After tires are manufactured at the production facilities, they are distributed through the distribution centers and the retailers to the end users. The location of retailers is fixed, and there might be unsatisfied demands due to uncertainty in demand. In the reverse flow, used tires are transferred to collection centers, where the initial inspection is performed, and based on the condition of the tires, they are either routed to retreading center or the recycling centers.

The main objective in the CLSC network design problem is to maximize the total profit of the supply chain by choosing the optimal number and location of facilities, their capacities, and the flow of products between the facilities, while reducing the environmental impacts and maximizing the social benefits. In real life, the product demand could be a function of economic condition; therefore, in this study, the future demand was expressed under a few scenarios. The return rates, retreading rates, recycling rates, procurement, and production costs are fuzzy; therefore, the possibilistic approach was utilized to deal with these parameters.

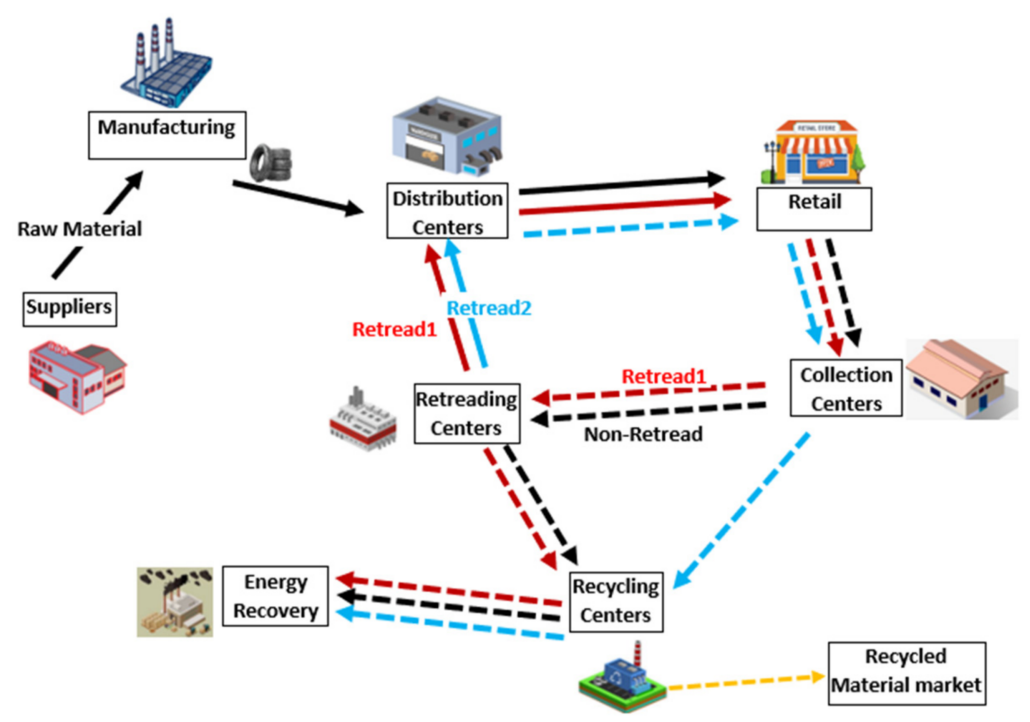

Figure 2. Schematic view of the proposed CLSC.

\subsection{Problem Assumption and Notation}

The main characteristics and assumptions are as follows:

- The potential location of new manufacturing centers, distribution centers, retreading centers, and recycling centers is fixed and predefined.

- Production, distribution, retreading, and recycling capacities are limited, and all are subject to capacity level $\mathrm{U}$.

- Capacity of the energy recovery centers is unlimited, but it has the most negative environmental impact.

- The return, recycling, and retreading rates of new and retread tires are fuzzy.

- Raw material procurement costs and production costs are fuzzy, and their fuzzy expected value are utilized.

- Customer demand is stochastic and is expressed in different scenarios.

- Ground transportation is the only transportation method used in the network.

- There is cost associated with the collection of used tires. This provides an incentive for the end users to return the EOL tires.

- Tires can be retreaded two times, retread-1 and retread-2, and each tire will be identified how many times it has been retreaded. 
- The value of the tires that were retreaded once (retread-1) is greater than the value of the tires that were retreaded two times (retread-2), and both are less than the value of the new tires.

- The demand for new tires must be satisfied, and there is a penalty for unsatisfied demand. There is no obligation to meet the demand for retread tires.

Table 2 shows the list of indices, parameters and variables related to the network under study.

Table 2. The list of model indices, parameters and variables.

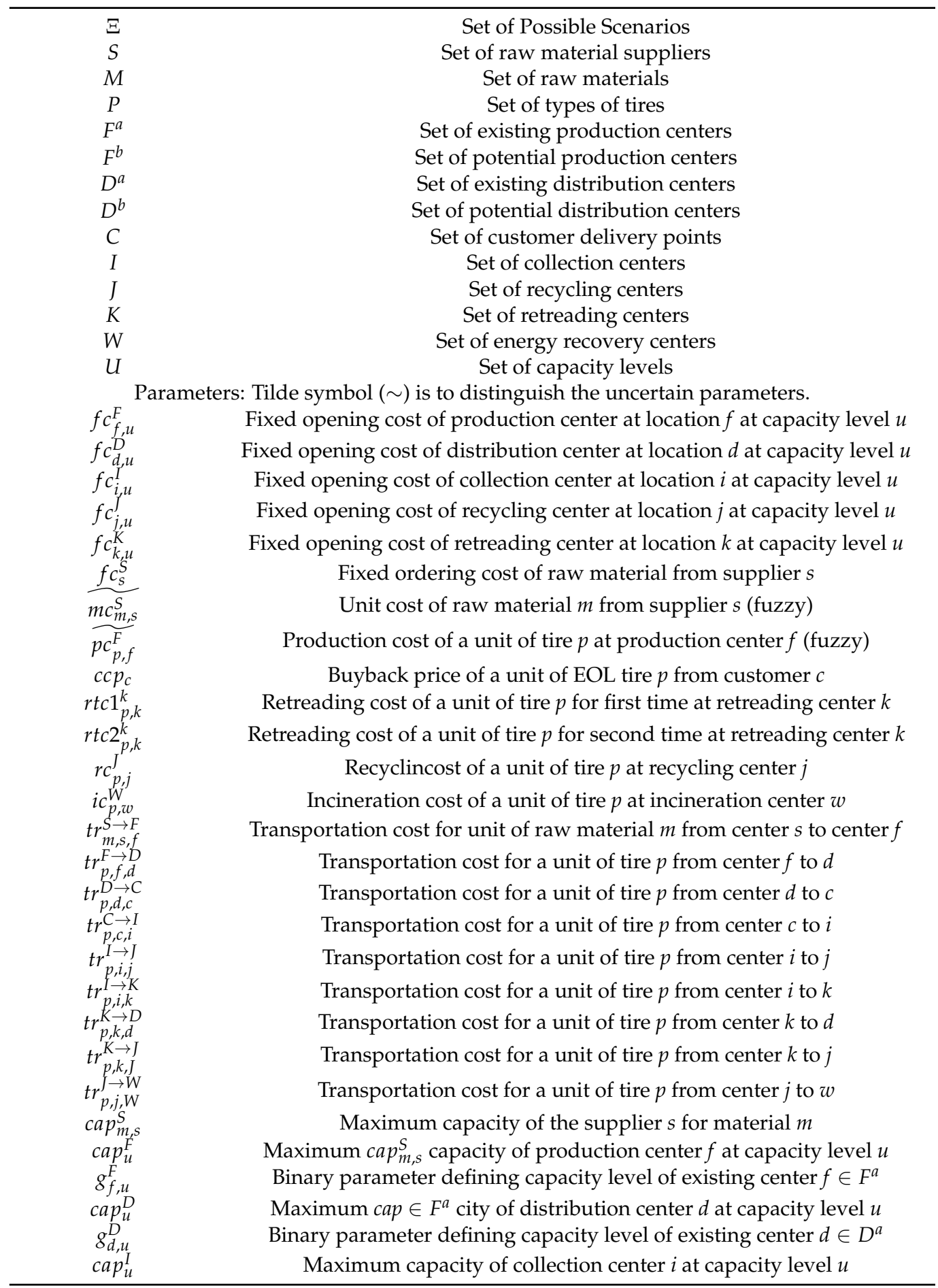


Table 2. Cont.

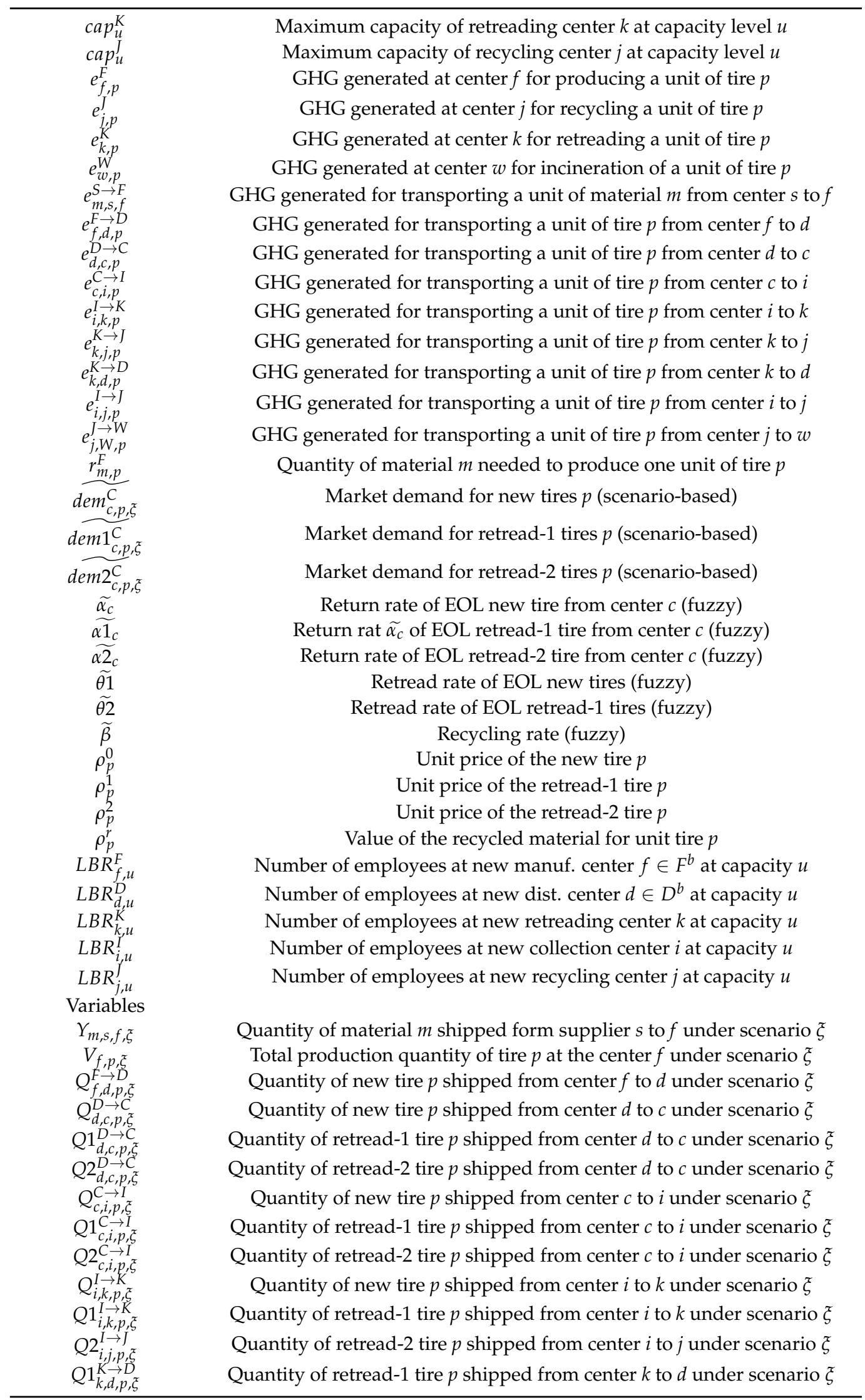


Table 2. Cont.

\begin{tabular}{cc}
\hline$Q 2_{k, d, p, \xi}^{K \rightarrow D}$ & Quantity of retread-2 tire $p$ shipped from center $k$ to $d$ under scenario $\xi$ \\
$Q 1_{k, j, p, \xi}^{K \rightarrow,}$ & Quantity of retread-1 tire $p$ shipped from center $k$ to $j$ under scenario $\xi$ \\
$Q 2_{k, j, p, \xi}^{K \rightarrow J}$ & Quantity of retread-2 tire $p$ shipped from center $k$ to $j$ under scenario $\xi$ \\
$Q_{j, p, \xi}^{J}$ & Total quantity of recycled tire $p$ at recycling center $j$ under scenario $\xi$ \\
$Q_{j, W, W, \xi}^{J \rightarrow W}$ & Quantity of tire $p$ shipped from center $j$ to $w$ under scenario $\xi$ \\
$Q_{w, p, \xi}^{W}$ & Total quantity $d \in D^{b} f$ tire $p$ incinerated at center $w$ under scenario $\xi$ \\
$x_{f, u}^{F}$ & Binary variables: \\
$x_{d, u}^{D}$ & Indicating if center $f$ at capacity level $u$ opened at location $f \in F^{b}$ or not \\
$x_{i, u}^{I}$ & Indicating if center $d$ at capacity level $u$ opened at location $d \in D^{b}$ or not \\
$x_{k, u}^{K}$ & Indicating if center $i$ at capacity level $u$ opened or not \\
$x_{j, u}^{J}$ & Indicating if center $k$ at capacity level $u$ opened or not \\
$x_{s}^{S}$ & Indicating if center $j$ at capacity level $u$ opened or not \\
\hline
\end{tabular}

\subsection{Problem Formulation}

In the following, we introduce three objective functions $z_{1}, z_{2}$, and $z_{3}$, under scenario $\xi$, representing the total profit of the network, the total environmental impacts, and the social benefits, respectively.

$$
\begin{aligned}
& \max Z_{1}=\left[\sum_{d} \sum_{c} \sum_{p}\left(\rho_{p}^{0} Q 1_{d, c . p, \xi^{\prime}}^{D \rightarrow C}+\rho_{p}^{1} Q 1_{d, c, p, \eta, \xi}^{D \rightarrow C}+\rho_{p}^{2} Q 2_{d, c, p, \xi}^{D \rightarrow C}\right)+\sum_{j} \sum_{p} \rho_{p}^{r} Q_{j, p, \xi}^{J}\right] \\
& -\left(\left[\sum_{f \in F^{b}} \sum_{u} f c_{f, u}^{F} \cdot x_{f, u}^{F}+\sum_{d \in D^{b}} \sum_{u} f c_{d, u}^{D} \cdot x_{d, u}^{D}+\sum_{i} \sum_{u} f c_{i, u}^{I} \cdot x_{i . u}^{I}\right.\right. \\
& \left.+\sum_{j} \sum_{u} f c_{j, u}^{J} \cdot x_{j, u}^{J}+\sum_{k} \sum_{u} f_{k, u}^{K} \cdot x_{k, u}^{K}\right] \\
& +\left[\sum_{s} f_{s}^{S} \cdot x_{s}^{S}+\sum_{m} \sum_{s} \sum_{f} \widetilde{m c_{m, S, \xi}^{S}} \cdot Y_{m, s, f, \xi}\right]+\left[\sum_{f} \underset{p}{\sum_{p} c_{p, f}^{F}} \cdot V_{f, p, \xi}\right] \\
& +\left[\sum_{c} \sum_{i} \sum_{p} c c_{p, c}\left(Q_{c, i, p, \xi}^{C \rightarrow I}+Q 1_{c, i, p, \xi}^{C \rightarrow I}+Q 2_{c, i, p, \xi}^{C \rightarrow I}\right)\right] \\
& +\left[\sum_{k} \sum_{d} \sum_{p}\left(r t c 1_{p, k}^{k} Q 1_{k, d, p, \xi}^{K \rightarrow D}+r t c 2_{p, k}^{k} Q 2_{k, d, p, \xi}^{K \rightarrow D}\right)\right]+\left[\sum_{j} \sum_{p} r c_{p j}^{J} Q_{j, p, \xi}^{J}\right] \\
& +\left[\sum_{w} \sum_{p} i c_{p, w}^{W} Q_{w, p, \xi}^{W}\right] \\
& +\left[\sum_{m} \sum_{s} \sum_{f} t r_{m, s, f}^{S \rightarrow F} \cdot Y_{m, s, f, \xi}+\sum_{f} \sum_{d} \sum_{p} \operatorname{tr} r_{p, f}^{F \rightarrow D} \cdot Q_{f, d, p, \xi}^{F \rightarrow D}\right. \\
& +\sum_{d} \sum_{c} \sum_{p} \operatorname{tr} \underset{p, d, c}{D \rightarrow C} \cdot\left(Q_{d, c, p, p, \xi}^{D \rightarrow C}+Q 1_{d, c, p, \eta, \xi}^{D \rightarrow C}+Q 2_{d, c, p, \xi}^{D \rightarrow C}\right) \\
& +\sum_{c} \sum_{i} \sum_{p} \operatorname{tr} r_{p, c, i}^{C \rightarrow I}\left(Q_{c, i, p, \xi}^{C \rightarrow I}+Q 1_{c, i, p, \xi}^{C \rightarrow I}+Q 2_{c, i, i, \xi}^{C \rightarrow I}\right) \\
& +\sum_{i} \sum_{k} \sum_{p} \operatorname{tr} r_{p, i, k}^{I \rightarrow K}\left(Q_{i, k, p, \xi}^{I \rightarrow K}+Q 1_{i, k, p, \xi}^{I \rightarrow K}\right)+\sum_{i} \sum_{j} \sum_{p} \operatorname{tr} \operatorname{tr}_{p, i, j}^{I \rightarrow J}\left(Q 2_{i, j, p, \xi}^{I \rightarrow I}\right)
\end{aligned}
$$

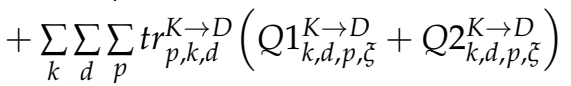

$$
\begin{aligned}
& +\sum_{k} \sum_{j} \sum_{p} \operatorname{tr}_{p, k, j}^{K \rightarrow J}\left(Q 1_{k, j, p, \xi}^{K \rightarrow J}+Q 2_{k, j, p, \xi}^{K \rightarrow J}\right) \\
& \left.\left.+\sum_{j} \sum_{w} \sum_{p} \operatorname{tr} \operatorname{tr}_{p, j, w}^{J \rightarrow W} \cdot Q_{j, W, p, \xi}^{J \rightarrow W}\right]\right) \quad \forall \xi \in \Xi
\end{aligned}
$$


Equation (1) maximizes the total profit of the supply chain. The components of the Equation (1) include the following: the overall cost of opening new centers, cost of procurement of raw material, overall manufacturing cost, collection cost, overall retreading cost, overall recycling cost, overall energy recovery cost, total transportation cost, revenue from sales of new tires, revenue from sales of tires that are retreaded once, revenue from sales of tires that are retreaded twice, and revenue from sales of recycled material.

$$
\begin{aligned}
& \min Z_{2}=\left[\sum_{f} \sum_{p} e_{f, p}^{F} . V_{f, p, \xi}\right]+\left[\sum_{k} \sum_{d} \sum_{p} e_{k, p}^{K}\left(Q 1_{k, d, p, \xi}^{K \rightarrow D}+Q 2_{k, d, p, \xi}^{K \rightarrow D}\right)\right] \\
& +\left[\sum_{j} \sum_{p} e_{j, p}^{J} Q_{j, p, \tau}^{J}\right]+\left[\sum_{w} \sum_{p} e_{w, p}^{W} Q_{w, p, \xi}^{W}\right] \\
& +\left[\sum_{m} \sum_{s} \sum_{f} \sum_{p} e_{m, s, f}^{S \rightarrow F} \cdot Y_{m, s, f, \xi}+\sum_{f} \sum_{d} \sum_{p} e_{p, f, d}^{F \rightarrow D} \cdot Q_{f, d}^{F \rightarrow, p}, \bar{f}\right. \\
& +\sum_{d} \sum_{c} \sum_{p} e_{p, d, c}^{D \rightarrow C}\left(Q_{d, c, p, p, \xi}^{D \rightarrow C}+Q 1_{d, c, p, p, \xi}^{D \rightarrow C}+Q 2_{d, c, p, \eta \xi}^{D \rightarrow C}\right) \\
& +\sum_{c} \sum_{i} \sum_{p} e_{p, c, i}^{C \rightarrow I}\left(Q_{c, i, p, \xi}^{C \rightarrow I}+Q 1_{c, i, p, \xi}^{C \rightarrow I}+Q 2_{c, i, p, \xi}^{C \rightarrow I}\right) \\
& +\sum_{i} \sum_{k} \sum_{p} e_{p, i, k}^{I \rightarrow K}\left(Q_{i, k, p, \xi}^{I \rightarrow K}+Q 1_{i, k, k, \xi}^{I \rightarrow K}\right) \\
& +\sum_{i} \sum_{j} \sum_{p} e_{p, i, j}^{I \rightarrow J}\left(Q 2_{i, j, p, \xi}^{I \rightarrow J}\right) \\
& +\sum_{k} \sum_{d} \sum_{p} e_{p, k, d}^{K \rightarrow D}\left(Q 1_{k, d, p, \xi}^{K \rightarrow D}+Q 2_{k, d, p, \xi}^{K \rightarrow D}\right) \\
& +\sum_{k} \sum_{j} \sum_{p} e_{p, k, j}^{K \rightarrow J}\left(Q 1_{k, j, p, \xi}^{K \rightarrow J}+Q 2_{k, j, p, \xi}^{K \rightarrow J}\right) \\
& \left.+\sum_{j} \sum_{W} \sum_{p} e_{p, j, k}^{J \rightarrow W} \cdot Q_{j, W, p, \xi}^{J \rightarrow W}\right] \quad \forall \xi \in \Xi
\end{aligned}
$$

Equation (2) minimizes the overall environmental impact, which is expressed as the amount of greenhouse gases (GHGs) generated at production centers, retreading centers, recycling centers, and energy recovery centers, as well as during the transportation of raw material and finished tires between centers. Among greenhouse gases, this research is focusing on carbon dioxide $\left(\mathrm{CO}_{2}\right)$ emission.

$$
\begin{gathered}
\max _{3}=\left[\sum_{f \in F^{b}} \sum_{u} L B R_{f, u}^{F} \cdot x_{f, u}^{F}+\sum_{d \in D^{b}} \sum_{u} L B R_{d, u}^{D} \cdot x_{d, u}^{D}+\sum_{i} \sum_{u} L B R_{i, u}^{I} \cdot x_{i . u}^{I}\right. \\
\left.+\sum_{j} \sum_{u} L B R_{j, u}^{J} \cdot x_{j, u}^{J}+\sum_{k} \sum_{u} L B R_{k, u}^{K} \cdot x_{k, u}^{K}\right] \forall \xi \in \Xi
\end{gathered}
$$

Equation (3) maximizes the number of job opportunities created as a result of expanding the forward logistics and also opening new facilities in reverse logistics.

Constraints:

$$
\begin{gathered}
\sum_{f} Y_{m, s, f, \xi} \leq c a p_{m, s}^{S} x_{s}^{S} ; \forall m \in M, s \in S, \xi \in \Xi \\
\sum_{p} V_{f, p, \xi} \leq \sum_{u} c a p_{u}^{F} \cdot g_{f, u}^{F} ; \forall f \in F^{a}, \xi \in \Xi \\
\sum_{p} V_{f, p, \xi} \leq \sum_{u} c a p_{u}^{F} \cdot x_{f, u}^{F} ; \forall f \in F^{b}, \xi \in \Xi \\
\sum_{u} x_{f, u}^{F} \leq 1 ; \forall f \in F^{b}, \xi \in \Xi \\
\sum_{p} r_{m}^{F} V_{f, p, \xi} \leq \sum_{s} Y_{m, s, f, \xi} ; \forall m \in M, f \in F, \xi \in \Xi
\end{gathered}
$$




$$
\begin{aligned}
& V_{f, p, \xi}=\sum_{d} Q_{f, d, p, \xi}^{F \rightarrow D} ; \forall f \in F, p \in P, \xi \in \Xi \\
& \sum_{f} \sum_{p} Q_{f, d, p, \xi}^{F \rightarrow D}+\sum_{k} \sum_{p}\left(Q 1_{k, d, p, \xi}^{K \rightarrow D}+Q 2_{k, d, p, \xi}^{K \rightarrow D}\right) \leq \sum_{u} c a p_{u}^{D} \cdot g_{d, u}^{D} ; \forall d \in D^{a}, \xi \in \Xi \\
& \sum_{f} \sum_{p} Q_{f, d, p, \xi}^{F \rightarrow D}+\sum_{k} \sum_{p}\left(Q 1_{k, d, p, \xi}^{K \rightarrow D}+Q 2_{k, d, p, \xi}^{K \rightarrow D}\right) \leq \sum_{u} c a p_{d}^{D} \cdot x_{d, u}^{D} ; \forall d \in D^{b}, \xi \in \Xi \\
& \sum_{u} x_{d, u}^{D} \leq 1 ; \forall d \in D^{b} \\
& \sum_{f} Q_{f, d, p, \xi}^{F \rightarrow D}=\sum_{c} Q_{d, c, p, \xi^{\prime}}^{D \rightarrow C} ; \forall d \in D, p \in P, \xi \in \Xi \\
& \sum_{k} Q 1_{k, d, p, \xi}^{K \rightarrow D}=\sum_{c} Q 1_{d, c, p, \xi}^{D \rightarrow C} ; \forall d \in D, p \in P, \xi \in \Xi \\
& \sum_{k} Q 2_{k, d, p, \xi}^{K \rightarrow D}=\sum_{c} Q 2_{d, c, p, \xi}^{D \rightarrow C} ; \forall d \in D, p \in P, \xi \in \Xi \\
& \sum_{d} Q_{d, c . p, \xi}^{D \rightarrow C}=\widetilde{\operatorname{dem}_{c, p, \xi}^{C}} ; \forall c \in C, p \in P, \xi \in \Xi \\
& \sum_{d} Q 1_{d, c, p, \xi}^{D \rightarrow C} \leq \widetilde{d e m 1_{c, p, \xi}^{C}} ; \forall c \in C, p \in P, \xi \in \Xi \\
& \sum_{d} Q 2_{d, c, p, \xi}^{D \rightarrow C} \leq d e m 2_{c, p, \xi}^{C} ; \forall c \in C, p \in P, \xi \in \Xi \\
& \sum_{i} Q_{c, i, p, \xi}^{C \rightarrow I}=\widetilde{\alpha_{c}} \sum_{d} Q_{d, c, p, \xi, \xi}^{D \rightarrow C} ; \forall c \in C, p \in P, \xi \in \Xi \\
& \sum_{i} Q 1_{c, i, p, \xi}^{C \rightarrow I^{\prime}}=\widetilde{\alpha 1_{c}} \sum_{d} Q 1_{d, c, p, \xi}^{D \rightarrow C} ; \forall c \in C, p \in P, \xi \in \Xi \\
& \sum_{i} Q 2_{c, i, p, \xi}^{C \rightarrow I}=\widetilde{\alpha 2_{c}} \sum_{d} Q 2_{d, c, p, \xi}^{D \rightarrow C} ; \forall c \in C, p \in P, \xi \in \Xi \\
& \sum_{c} \sum_{p} Q_{c, i, p, \xi}^{C \rightarrow I}+\sum_{c} \sum_{p} Q 1_{c, i, p, \xi}^{C \rightarrow I}+\sum_{c} \sum_{p} Q 2_{c, i, p, \xi}^{C \rightarrow I} \leq \sum_{u} c a p_{i}^{I} \cdot x_{i, u}^{I} ; \forall i \in I, \xi \in \Xi \\
& \sum_{c} Q_{c, i, p, \xi}^{C \rightarrow I}=\sum_{k} Q_{i, k, p, \xi}^{I \rightarrow K} ; \forall i \in I, p \in P, \xi \in \Xi \\
& \sum_{c} Q 1_{c, i, p, \xi}^{C \rightarrow I}=\sum_{k} Q 1_{i, k, p, \xi}^{I \rightarrow K} ; \forall i \in I, p \in P, \xi \in \Xi \\
& \sum_{c} Q 2_{c, i, p, \xi}^{C \rightarrow I}=\sum_{j} Q 2_{i, j, p, \xi^{\prime}}^{I \rightarrow \rightarrow} ; \forall i \in I, p \in P, \xi \in \Xi \\
& \sum_{i} \sum_{p}\left(Q_{i, k, p, \xi}^{I \rightarrow K}+Q 1_{i, k, p, \xi}^{I \rightarrow K}\right) \leq \sum_{u} c a p_{u}^{K} \cdot x_{k, u}^{K} ; \forall k \in K, \xi \in \Xi \\
& \sum_{d} Q 1_{k, d, p, \xi}^{K \rightarrow D}=\widetilde{\theta 1} \sum_{i} Q_{i, k, p, \xi}^{I \rightarrow K} ; \forall k \in K, p \in P, \xi \in \Xi \\
& \sum_{d} Q 2_{k, d, p, \xi}^{K \rightarrow D}=\widetilde{\theta 2} \sum_{i} Q 1_{i, k, p, \tau}^{I \rightarrow K} ; \forall k \in K, p \in P, \xi \in \Xi \\
& \sum_{j} Q 1_{k, j, p, \xi}^{K \rightarrow J}=\sum_{i} Q_{i, k, p, \xi}^{I \rightarrow K}-\sum_{d} Q 1_{k, d, p, \xi}^{K \rightarrow D} ; \forall k \in K, p \in P, \xi \in \Xi \\
& \sum_{j} Q 2_{k, j, p, \xi}^{K \rightarrow I}=\sum_{i} Q 1_{i, k, p, \xi}^{I \rightarrow K}-\sum_{d} Q 2_{k, d, p, \xi}^{K \rightarrow D} ; \forall k \in K, p \in P, \xi \in \Xi \\
& \sum_{k} \sum_{p} Q 1_{k, j, p, \xi}^{K \rightarrow J}+\sum_{k} \sum_{p} Q 2_{k, j, p, \xi}^{K \rightarrow J}+\sum_{i} \sum_{p} Q 2_{i, j, p, \xi}^{I \rightarrow J} \leq \sum_{u} c a p_{j}^{J} \cdot x_{j, u}^{J} ; \forall j \in J, \xi \in \Xi
\end{aligned}
$$




$$
\begin{gathered}
Q_{j, p, \xi}^{J}=\widetilde{\beta}\left(\sum_{k} Q 1_{k, j, p, \xi}^{K \rightarrow J}+\sum_{k} Q 2_{k, j, p, \xi}^{K \rightarrow J}+\sum_{i} Q 2_{i, j, p, \xi}^{I \rightarrow J}\right) ; \forall j \in J, p \in P, \xi \in \Xi \\
\sum_{w} Q_{j, w, p, \xi}^{J \rightarrow W}=\sum_{k} Q 1_{k, j, p, \xi}^{K \rightarrow J}+\sum_{k} Q 2_{k, j, p, \xi}^{K \rightarrow J}+\sum_{i} Q 2_{i, j, p, \xi}^{I \rightarrow J}-Q_{j, p, \xi}^{I} ; \forall j \in J, p \in P, \xi \in \Xi \\
Q_{w, p, \xi}^{W}=\sum_{j} Q_{j, w, p, \xi}^{J \rightarrow W} ; \forall w \in W, p \in P, \xi \in \Xi \\
\left\{\begin{array}{c}
x_{(*, *)}^{(.)}, Y_{m, s, f, \xi} \in\{0,1\} \\
V_{f, p, \xi}, Q_{(*, *)}^{-\rightarrow .} \geq 0
\end{array}\right.
\end{gathered}
$$

Constraint (4) ensures that the total quantity of raw material sent to tire manufacturing centers is less than or equal to the maximum capacity of each supplier for that raw material. Constraints (5) and (6) ensure that the total quantity of tires produced at each manufacturing center is less than or equal to the maximum capacity of each existing and potential manufacturing center. Constraint (7) ensures that only one production facility at capacity level $\mathrm{u}$ can be open at location $\mathrm{f}$. Constraint (8) guarantees that the raw material needed at production centers are satisfied. Constraint (9) is an equilibrium constraint indicating the total quantity of tires transferred from each manufacturing center $\mathrm{f}$ to distribution center $\mathrm{d}$ is equal to the quantity of tires manufactured at that center. Constraints (10) and (11) ensure that the maximum capacity of the existing and potential distribution centers are not violated. Constraint (12) guarantees that only one distribution center at capacity level $u$ can be open at any potential location d. Constraints (13)-(15) are equilibrium constraints to ensure the total quantity of new and retread (retread- 1 and retread-2) tires transferred out of each distribution center is equal to the total quantity of tires enter that center. Constraint (16) ensures that the market demand for new tires is satisfied. Constraints (17) and (18) indicate that the demand for retread-1 and retread-2 tires does not have to be entirely satisfied. It should be noted that the demand parameters are stochastic-scenario-based. Constraints (19)-(21) calculate the total quantity of tires returned to each collection center. It should be noted $\widetilde{\alpha}_{c}, \widetilde{\alpha 1_{c}}, \widetilde{\alpha 2_{c}}$ are fuzzy parameters that will be discussed in the next section. Constraint (22) controls the maximum capacity of the collection centers. Constraints (23)-(25) are equilibrium constraints which create flow balance at each collection center. Constraint (26) ensures the flow of products into and out of each retreading center do not exceed the capacity of that center. Constraints (27) and (28) calculate the quantity of the tires that are being successfully retreaded at each retreading center. Moreover, $(\widetilde{\theta 1})$ and $(\widetilde{\theta 2})$ are fuzzy parameters. Constraints (29) and (30) calculate the total quantity of new and retread tires that cannot be retreaded at retreading centers and are being transferred to recycling centers. Constraint (31) controls the capacity of the recycling centers. Constraint (32) calculates the total quantity of products transfers to each recycling center. It should be noted that $\widetilde{\beta}$ is a fuzzy parameter. Constraint (33) creates equilibrium at recycling centers. Constraint (34) calculates the total number of tires being incinerated at each energy recovery center. Ultimately, constraint (35) enforces the binary and non-negative constraints on the corresponding decision variables.

The proposed tire CLSC model is a Mixed Integer Linear Programming (MILP) problem under hybrid uncertainty. Fuzzy uncertainty related to return rate, retreading rate, recycling rate, procurement cost, production cost, and scenario-based uncertainty related to demand parameters. In the following, we discuss a method that can simultaneously deal with these hybrid uncertainties.

\section{The Proposed Approach}

Robust Fuzzy/Possibilistic Stochastics Programming (RFSP) is a novel approach in mathematical programming that deals with hybrid uncertain parameters [6]. It is an expansion of possibilistic programing [43] in which the possible scenarios of the disturbance in the system are additionally being considered and addressed using stochastic scenario- 
based programming method [42]. An example of a disturbance in the system would be a change in customer behavior that creates a noticeable change in demand in comparison to the initial forecast. These changes can create a new scenario in the system with respect to demand parameters.

Since the proposed tire CLSC problem faces both scenario-based uncertainty related to demand parameters and fuzzy uncertainty associated with return rates, retreading rates, recycling rates, procurement, and production costs, Robust Fuzzy/Possibilistic Stochastics Programming method is proposed in this research to deal with hybrid uncertain parameters.

In the following, we first describe the basics of robust stochastic programming and possibilistic programming. Then, the proposed RFSP approach is explained; and at the end, this approach is used to control the uncertainties in the proposed tire CLSC problem.

\subsection{Stochastic Programming}

In general, the scenario-based stochastic programming approach can be described as follows:

$$
\left\{\begin{array}{c}
\max \sum_{\xi \in \Xi} \pi_{\xi} . z_{\xi} \\
z_{\xi}=c_{\xi}^{\top} \cdot x_{\xi}+d_{\xi}^{\top} y \quad \forall \xi \in \Xi \\
A_{\xi} x_{\xi}+K_{\xi} y \leq b_{\xi} \quad \forall \xi \in \Xi \\
R y=q \\
y \in Y, x_{\xi} \geq 0
\end{array}\right.
$$

where $z_{\xi}$ is the objective function under scenario $\xi \in \Xi, x_{\xi}$ are the scenario dependent variables, $y$ are the variables independent of the scenario (i.e., binary variables), $\left(c_{\xi}, d_{\xi}, A_{\tilde{\zeta}}, K_{\tilde{\zeta}}, b_{\xi}\right)$ are the parameters under scenario $\xi \in \Xi,(R, q)$ are crisp parameters and $\pi_{\xi}$ is the probability of occurrence of scenario $\xi \in \Xi[7,73]$.

Since scenario-based programming models are not sensitive to deviations of the value of the objective function, in order to prevent excessive deviation of the of the objective function under different scenarios, the variance of the objective function is also considered. Therefore, the resulting robust stochastic programming model would be as follows:

$$
\left\{\begin{array}{c}
\max \sum_{\xi \in \Xi} \pi_{\xi} . z_{\xi}-\lambda \cdot \sum_{\xi \in \Xi} \pi_{\xi}\left(2 \theta_{\xi}-\left(z_{\xi}-\sum_{\xi^{\prime} \in \Xi} \pi_{\xi} . z_{\xi^{\prime}}\right)\right)-\omega \sum_{\xi \in \Xi} \pi_{\xi} . \Delta_{\xi} \\
z_{\xi}=c_{\xi}^{\top} \cdot x_{\xi}+d_{\xi}^{\top} y \forall \xi \in \Xi \\
\theta_{\xi} \geq z_{\xi}-\sum_{\xi \in S} \pi_{\xi} . z_{\xi} \forall \xi \in \Xi \\
A_{\xi} x_{\xi}+K_{\xi} y \leq b_{\xi}+\Delta_{\xi} \quad \forall \xi \in \Xi \\
R y=q \\
y \in Y, x_{\xi} \geq 0 \\
\theta_{\xi} \geq 0
\end{array}\right.
$$

where $\lambda$ denotes the importance or weighing scale to measure the tradeoff between optimality and the cost under different scenarios. $\theta_{\xi}$ denotes the absolute deviation from the mean under each scenario. $\Delta_{\xi}$ represent the deviation for violation of the control constraint, and $\omega$ is the robustness coefficient or feasibility robustness multiplier which its value can be equal to the penalty cost of the shortage. It should be noted that the above model is known as the Mulvey Robust stochastic model [42], although the above model is the linear version of the model based on the research of Yu and Li [7]. 
In the above model, the following linearization was utilized:

$$
\min \sum_{\xi \in \Xi} \pi_{\xi}\left|z_{\xi}-\sum_{\xi^{\prime} \in \Xi} \pi_{\xi^{\prime}} \cdot z_{\xi^{\prime}}\right| \Longleftrightarrow \text { Linearization }\left\{\begin{array}{c}
\min \sum_{\xi^{\prime} \in \Xi} \pi_{\xi}\left(2 \theta_{\xi}-\left(z_{\xi}-\sum_{\xi^{\prime} \in S} \pi_{\xi^{\prime}} \cdot z_{\xi^{\prime}}\right)\right) \\
\theta_{\xi} \geq z_{\xi}-\sum_{\xi \in \Xi} \pi_{\xi} . z_{\xi} \forall \xi \in \Xi
\end{array}\right.
$$

\subsection{Possibilistic Programming}

If some of the data in an optimization problem are imprecise due to insufficient data or unavailability of the information, but their approximate values can be expressed in fuzzy numbers, fuzzy mathematical programming can be used [74]. If fuzzy data in the optimization problem are expressed as Trapezoidal Fuzzy Number (TFN) and the possibility of occurrence of the possibilistic parameters are measured by Pos. and Nec., then possibilistic programming can be used, where Pos. is the maximum level of occurrence of parameters and Nec. is the minimum possibility level.

The general form of possibilistic programming can be expressed as follows:

$$
\left\{\begin{array}{c}
\max Z=E(\widetilde{\mathcal{C}} x) \\
\text { s.t. } \\
\operatorname{Me}(\operatorname{Ax} \leq \widetilde{\mathcal{F}}) \geq \mathbb{P} \\
x \in X
\end{array}\right.
$$

where $\mathrm{Me}($.$) is a fuzzy measure; \widetilde{\mathcal{F}}=\left(f_{(1)}, f_{(2)}, f_{(3)}, f_{(4)}\right)$ and $\widetilde{c}=\left(c_{(1)}, c_{(2)}, c_{(3)}, c_{(4)}\right)$ are the fuzzy parameters in the form of TFN, Figure $3 ; \mathbb{P}$ is the minimum confidence level; and $E(\widetilde{c} x)$ is the fuzzy expected value of the objective function, which can be expressed as follows:

$$
E(\widetilde{c} x)=\frac{\left(c_{(1)}+2 c_{(2)}+2 c_{(3)}+c_{(4)}\right)}{6} x
$$

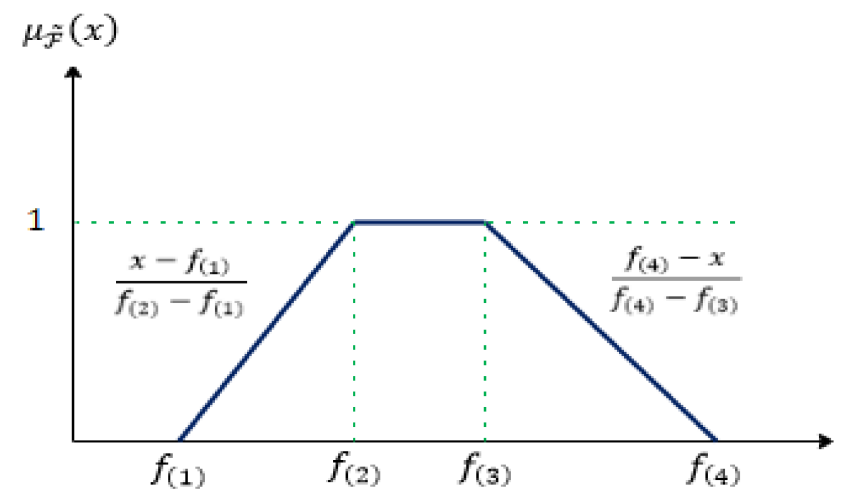

Figure 3. Function of Trapezoidal Fuzzy Numbers.

In order to control fuzzy constraints, one of the most widely used fuzzy measures is the credibility measure ( $\mathrm{Cr}$ ), which is expressed as the average of the optimistic (Pos) and pessimistic (Nec) measures:

$$
\mathrm{Cr}(.)=\frac{1}{2}(\operatorname{Pos}(.)+\mathrm{Nec}(.))
$$

And the following relationship can exist [70]:

$$
\left\{\begin{array}{l}
\operatorname{Cr}(\mathrm{Ax} \geq \widetilde{\mathcal{F}}) \geq \mathbb{P} \Longleftrightarrow \mathrm{Ax} \geq f_{(3)}+(2 \alpha-1)\left(f_{(4)}-f_{(3)}\right): \mathbb{P} \geq 0.5 \\
\operatorname{Cr}(\mathrm{Ax} \leq \widetilde{\mathcal{F}}) \geq \mathbb{P} \Longleftrightarrow \mathrm{Ax} \leq f_{(2)}-(2 \alpha-1)\left(f_{(2)}-f_{(1)}\right): \mathbb{P} \geq 0.5
\end{array}\right.
$$


One of the disadvantages of classical programming is that the $\mathbb{P}$ value is determined subjectively by the decision-maker (DM) and is usually set inactively or ultimately interactively and may not be optimal. This issue was first addressed in the research conducted by Pishvaee (2012) [43].

In their proposed model, taking into account the two concepts of feasibility robustness and optimality robustness, the optimal levels of $\mathbb{P}$ value are obtained proactively and objectively. This approach has been used in several studies [74-80].

\subsection{Robust Fuzzy/Possibilistic Stochastic Programming}

The RFSP approach is an extension of the combination of scenario-based stochastic programming and possibilistic programming [43] in which random scenarios and fuzzy data are controlled simultaneously [6]. The RFSP approach can be summarized as follows:

$$
\left\{\begin{array}{c}
\max \sum_{\xi \in \Xi} \pi_{\xi} \cdot z_{\xi}-\lambda \cdot \sum_{\xi \in \Xi} \pi_{\xi}\left(2 \theta_{\xi}-\left(z_{\xi}-\sum_{\xi^{\prime} \in \Xi} \pi_{\xi^{\prime}} \cdot z_{\xi^{\prime}}\right)\right)-\omega \sum_{\xi \in \Xi} \pi_{\xi} \cdot \Delta_{\xi}-\Psi . \vartheta(\mathbb{P}) \\
z_{\xi}=E\left(c_{\xi}^{\top} \cdot x_{\xi}+d_{\xi}^{\top} y\right)=\frac{\left(c_{(1)}+2 c_{(2)}+2 c_{(3)}+c_{(4)}\right)}{6} x_{\xi}+d_{\xi}^{\top} y \forall \xi \in \Xi \\
A_{\xi} x_{\xi}+K_{\xi} y \leq b_{(2) \xi}-(2 \mathbb{P}-1)\left(b_{(2) \xi}-b_{(1) \xi}\right)+\Delta_{\xi} \quad \forall \xi \in \Xi \\
R y=q \\
\theta_{\xi} \geq z_{\xi}-\sum_{s \in S} \pi_{\xi} \cdot z_{\xi} \forall \xi \in \Xi \\
y \in Y, x_{\xi} \geq 0 \\
\theta_{\xi} \geq 0 \\
\mathbb{P} \geq 0.5
\end{array}\right.
$$

where $\Psi$ is the coefficient for estimating fuzzy numbers, $\vartheta(\mathbb{P})$ is a non-negative descending function that calculates the violation of fuzzy constraints with confidence level $\mathbb{P}$. Moreover, $\vartheta(\mathbb{P})$ can be expressed as the difference between the substituted crisp numbers, $\left(b_{(2) \xi}-(2 \mathbb{P}-1)\left(b_{(2) \xi}-b_{(1) \xi}\right)\right)$, and the worst-case value of the imprecise parameters (TFNs) can be expressed as follows:

$$
\begin{aligned}
& \vartheta(\mathbb{P})=\left(b_{(2) \xi}-(2 \mathbb{P}-1)\left(b_{(2) \xi}-b_{(1) \xi}\right)\right)-b_{(1) \xi} \\
= & \left(b_{(2) \xi}-b_{(1) \xi}\right)-(2 \mathbb{P}-1)\left(b_{(2) \xi}-b_{(1) \xi}\right) \\
= & (1-2 \mathbb{P}+1)\left(b_{(2) \xi}-b_{(1) \xi}\right)=2(1-\mathbb{P})\left(b_{(2) \xi}-b_{(1) \xi}\right)
\end{aligned}
$$

$\Delta_{\xi}$ calculates shortage under each scenario. It should be noted that $\lambda$ denotes the weighing scale to measure the tradeoff between optimality and the cost under different scenarios, $\theta_{\xi}$ denotes the absolute deviation from the mean under each scenario. $\Delta_{\zeta}$ represents the deviation for violation of the control constraint, and $\omega$ is the model robustness coefficient or feasibility robustness multiplier.

\subsection{Coping with Multi-Objective Functions}

In order to solve multi-objective decision-making (MODM) optimization problems, several approaches have been proposed, such as Weighted Sum Method (WSM), Epsilon Constraint (EC), Augmented Epsilon Constraint (AEC), Goal Programing (GP), Lexicographic (Lex), and so on. The general form of a MODM problem is as follows:

$$
\left\{\begin{array} { c } 
{ \operatorname { M i n } ( f _ { 1 } ( x ) , f _ { 2 } ( x ) , \ldots , f _ { n } ( x ) ) } \\
{ x \in X }
\end{array} \left\{\begin{array}{c}
\operatorname{Min}\left(f_{1}(x), f_{2}(x), \ldots, f_{n}(x)\right) \\
x \in X
\end{array}\right.\right.
$$




\subsection{Epsilon Constraint}

Let us assume the first objective function is the main objective, and the remaining objectives will have an upper bound of epsilon and will be presented as constraints of the model. In this case, the EC method is being used, and the resulting single objective model can be described as follows:

$$
\left\{\begin{array}{c}
\operatorname{Min} f_{1}(x) \\
f_{i}(x) \leq e_{i} i=2,3, \ldots, n \\
x \in X
\end{array}\right.
$$

In the above model, the first objective is considered as the main objective, and the remaining objectives are considered as constraints and are bound by maximum $e_{i}$. By changing the $e_{i}$, different results can be obtained, which may be weakly efficient or not efficient.

This issue can be resolved with minor modification, which is known as the AEC method [81]. To better implement the AEC method, the appropriate range of epsilons $e_{i}$ can be obtained by using the Lex method [82]. In the AEC method, the appropriate range of $e_{i}$ is first determined and then the Pareto front obtained for different values of $e_{i}$.

\subsection{Appropriate Interval for $e_{i}$ with Lex Method}

In order to find the appropriate interval for $e_{i}$ related to the objective function $f_{i}(i=2, \ldots, n)$, the following optimization problem is first being solved:

$$
\operatorname{PayOff}_{j j}=\underset{x \in X}{\operatorname{Min} f_{j}(x) \quad j=1,2, \ldots, n}
$$

where PayOff $f_{j j}=f_{j}\left(x^{j, *}\right)$ is the optimum value of $f_{j}$ and $x^{j, *}$ is the vector of decision variables that optimizes $f_{j}$.

Then, with the solution that optimizes the objective function $f_{j}$, the value of the remaining objective functions is obtained as follows:

$$
\begin{gathered}
\text { PayOff }_{i j}=\operatorname{Min} f_{i}(x) \\
f_{j}(x)=\text { PayOff }_{j j} \\
x \in X \\
j=1,2, \ldots, n ; j \neq i
\end{gathered}
$$

where $\operatorname{PayOff} f_{j j}=f_{i}\left(x^{i, j, *}\right)$ is the optimum value of $f_{i}$ and $x^{i, j, *}$ is the vector of decision variables that optimizes $f_{i}$. Using the Lex method, the following payoff matrix is obtained:

$$
\text { PayOff }=\left[\text { payOff }_{i j}\right]
$$

After calculating the payoff table for $f_{i} i=1, \ldots, n$, the following can be defined:

$$
\begin{aligned}
& \operatorname{Min}\left(f_{i}\right)=\operatorname{Min}_{j}\left\{\text { payOff }_{i j}\right\}=\operatorname{payOff}_{i i} \\
& \operatorname{Max}\left(f_{i}\right)=\operatorname{Max}_{j}\left\{\operatorname{payOff}_{i j}\right\} \\
& R\left(f_{i}\right)=\operatorname{Max}\left(f_{i}\right)-\operatorname{Min}\left(f_{i}\right)
\end{aligned}
$$

Using the above definition, the appropriate rage for each $e_{i}$ can be obtained by using the Lex method:

$$
e_{i} \in\left[\operatorname{Min}\left(f_{i}\right), \operatorname{Max}\left(f_{i}\right)\right]
$$

The value $R\left(f_{i}\right)$ is used to normalize the objectives in the AEC objective functions. 


\subsection{Augmented Epsilon Constraint}

The AEC model can be expressed as follows:

$$
\left\{\begin{array}{c}
\operatorname{Min} f_{1}(x)-\sum_{i=2}^{n} \phi_{i} s_{i} \\
f_{i}(x)+s_{i}=e_{i} i=2,3, \ldots, n \\
x \in X \\
s_{i} \geq 0
\end{array}\right.
$$

where $s_{i}$ are non-negative variables, $\phi_{i}$ is a parameter for normalizing the first objective with respect to $f_{i}$ and $\phi_{i}=\frac{R\left(f_{1}\right)}{R\left(f_{i}\right)}$.

In the proposed AEC method in this study, first $e_{i} \in\left[\operatorname{Min}\left(f_{i}\right), \operatorname{Max}\left(f_{i}\right)\right]$ is obtained using Lex method, then Equation (45) is solved, which provides an optimum solution.

\section{Computational Experiment}

To check the validity, practicality, and performance of the model, this model was applied for the design and optimization of a network in Northeast USA, as illustrated in Figure 4 . The relevant data presented in Tables 3-6 were generated from the literature, field data, interviewing experts in the field, and some organizations involved in this field, including the EPA, US Tire Manufacturers Association, Tire Retread \& Repair Information Bureau (TRIB), Modern Tire Dealer (MTD), and Institute of Scrap Recycling Industries (ISRI).

In this research, there are ten types of medium/heavy truck tires considered. There are five groups of raw materials (synthetic polymers, natural rubber, steel, fillers, and curing compounds) required to manufacture a tire and five suppliers to purchase the raw material from, which are all located out of the state. There are also two existing production facilities and five distribution centers at medium-capacity level, two energy recovery centers, and forty commercial retail locations. We are considering the feasibility of expanding the network by adding two additional manufacturing facilities, five additional distribution centers, ten collection centers, four retreading centers, and five recycling centers. We believe that no tire should end up in the landfills, and by providing appropriate incentives, we can encourage customers to return the EOL tires for collection and processing. These facilities are selected such that they are in the proximity of the facilities they are linked to in the network flow and also to provide maximum coverage to the entire region. The goal is to optimize the number, location, and capacity of the facilities, increase the efficiency and profitability of the network and reduce the amount of greenhouse gases generated in the network while maximizing the total number of jobs that can be created. The demand for truck tires depends on the performance of the logistics and transportation industry in the country, which is directly correlated to the condition of the economy. We assume three scenarios for future demand, corresponding to demand at the time of poor economy (recession), during a good steady economy, and when the economy is growing. Ground transportation is the only mean of transferring the tires and raw material between the facilities. Assuming a 22-foot full truckload on each transfer, the cost of transferring one unit of tire per kilometer is $\$ 0.02$ and $\$ 0.002$ for one unit of raw material. The amount of $\mathrm{Co} 2$ generated per unit of tire transferred between facilities, using ground transportation is 0.002 kilogram per kilometer and is $0.0002 \mathrm{~kg}$ per kilometer for one unit of raw material being transferred. 


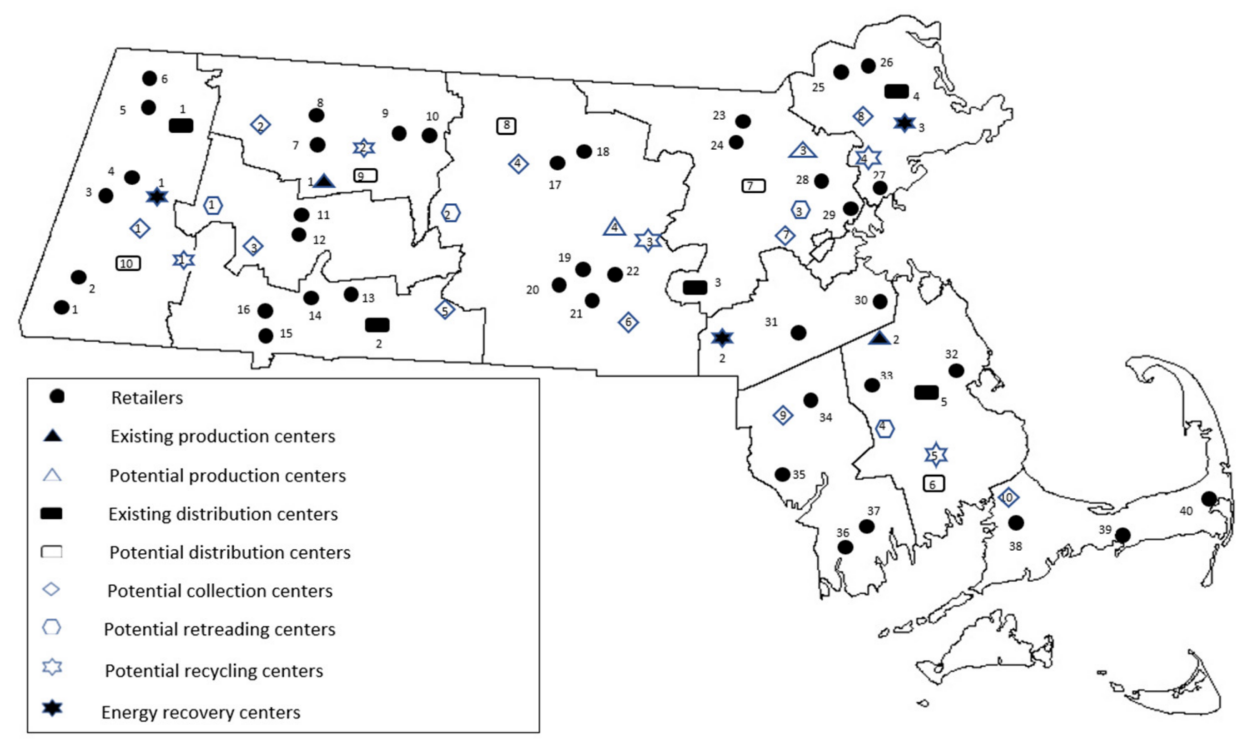

Figure 4. Location of the existing and potential facilities.

Table 3. Values for scenario-based parameters (demands).

\begin{tabular}{cccc}
\hline Scenarios $(\xi)$ & Recession & Good & Growing \\
\hline Scenario Probability & 0.20 & 0.50 & 0.30 \\
\hline$\widetilde{d e m_{c, p, \xi}^{C}}$ & $\mathrm{U}(5000-5100)$ & $\mathrm{U}(5100-5300)$ & $\mathrm{U}(5300-5500)$ \\
$\widetilde{d e m 1_{c, p, \xi}^{C}}$ & $\mathrm{U}(2400-2700)$ & $\mathrm{U}(2700-2800)$ & $\mathrm{U}(2800-2900)$ \\
$\widetilde{d e m 2_{c, p, \xi}^{C}}$ & $\mathrm{U}(500-600)$ & $\mathrm{U}(600-700)$ & $\mathrm{U}(700-800)$ \\
\hline
\end{tabular}

Table 4. Values for fuzzy parameters.

\begin{tabular}{|c|c|c|c|c|}
\hline Parameters & $\boldsymbol{c}_{(1)}$ & $c_{(2)}$ & $\boldsymbol{c}_{(3)}$ & $\boldsymbol{c}_{(4)}$ \\
\hline$\widetilde{\alpha_{c}}$ & $\mathrm{U}(0.7-0.75)$ & $\mathrm{U}(0.75-0.8)$ & $\mathrm{U}(0.80-0.85)$ & $\mathrm{U}(0.85-0.9)$ \\
\hline$\widetilde{\alpha 1_{c}}$ & $\mathrm{U}(0.65-0.7)$ & $\mathrm{U}(0.7-0.75)$ & $\mathrm{U}(0.75-0.8)$ & $\mathrm{U}(0.8-0.85)$ \\
\hline$\widetilde{\alpha 2_{c}}$ & $\mathrm{U}(0.55-0.6)$ & $\mathrm{U}(0.6-0.65)$ & $\mathrm{U}(0.65-0.7)$ & $\mathrm{U}(0.7-0.75)$ \\
\hline$\widetilde{\theta 1}$ & $\mathrm{U}(0.6-0.65)$ & $\mathrm{U}(0.65-0.7)$ & $\mathrm{U}(0.7-0.75)$ & $\mathrm{U}(0.75-0.8)$ \\
\hline$\widetilde{\theta 2}$ & $\mathrm{U}(0.5-0.55)$ & $\mathrm{U}(0.55-0.6)$ & $\mathrm{U}(0.6-0.65)$ & $\mathrm{U}(0.65-0.7)$ \\
\hline$\widetilde{\beta}$ & $\mathrm{U}(0.88-0.91)$ & $\mathrm{U}(0.91-0.93)$ & U (093-0.96) & $\mathrm{U}(0.96-0.99)$ \\
\hline$\widetilde{p c_{p, f}^{F}}$ & $\mathrm{U}(\$ 100-\$ 110)$ & $\mathrm{U}(\$ 110-\$ 120)$ & $\mathrm{U}(\$ 120-\$ 130)$ & $\mathrm{U}(\$ 130-\$ 140)$ \\
\hline$\overline{m c_{m=1, s}^{S}}$ & $\mathrm{U}(\$ 9, \$ 9.5)$ & $\mathrm{U}(\$ 9.5, \$ 10)$ & $\mathrm{U}(\$ 10, \$ 10.5)$ & $\mathrm{U}(\$ 10.5, \$ 11)$ \\
\hline$m c_{m=2, s}^{S}$ & $\mathrm{U}(\$ 43, \$ 44)$ & $\mathrm{U}(\$ 44, \$ 45)$ & $\mathrm{U}(\$ 45, \$ 46)$ & $\mathrm{U}(\$ 46, \$ 47)$ \\
\hline$\overline{m c_{m=3, s}^{S}}$ & $\mathrm{U}(\$ 9, \$ 9.5)$ & U (\$9.5-\$10) & $\mathrm{U}(\$ 10-\$ 10.5)$ & $\mathrm{U}(\$ 10.5-\$ 11)$ \\
\hline$\widehat{m c_{m=4, s}^{S}}$ & $\mathrm{U}(\$ 9, \$ 9.5)$ & $\mathrm{U}(\$ 9.5-\$ 10)$ & $\mathrm{U}(\$ 10-\$ 10.5)$ & $\mathrm{U}(\$ 10.5 \mathrm{k}-\$ 11)$ \\
\hline$m c_{m=5, s}^{S}$ & $\mathrm{U}(\$ 10, \$ 10.5)$ & $\mathrm{U}(\$ 10.5, \$ 11)$ & $\mathrm{U}(\$ 11, \$ 11.5)$ & $\mathrm{U}(\$ 11.5, \$ 12)$ \\
\hline
\end{tabular}


Table 5. Parameters at multi-capacity levels.

\begin{tabular}{|c|c|c|c|}
\hline \multicolumn{4}{|c|}{ Capacity Level (U) } \\
\hline & Low & Medium & High \\
\hline$f c_{f, u}^{F}$ & $\mathrm{U}(\$ 3 \mathrm{M}-\$ 4 \mathrm{M})$ & $\mathrm{U}(\$ 4 \mathrm{M}-\$ 6 \mathrm{M})$ & $\mathrm{U}(\$ 6 \mathrm{M}-\$ 10 \mathrm{M})$ \\
\hline$f c_{d u}^{D}$ & $\mathrm{U}(\$ 0.2 \mathrm{M}-\$ 0.3 \mathrm{M})$ & $\mathrm{U}(\$ 0.3 \mathrm{M}-\$ 0.5 \mathrm{M})$ & $\mathrm{U}(\$ 0.5 \mathrm{M}-\$ 0.7 \mathrm{M})$ \\
\hline$f c_{\mathrm{iu}}^{I}$ & $\mathrm{U}(\$ 0.2 \mathrm{M}-\$ 0.3 \mathrm{M})$ & $\mathrm{U}(\$ 0.3 \mathrm{M}-\$ 0.4 \mathrm{M})$ & $\mathrm{U}(\$ 0.4 \mathrm{M}-\$ 0.5 \mathrm{M})$ \\
\hline$f c_{j, u}^{J}$ & $\mathrm{U}(\$ 0.3 \mathrm{M}, \$ 0.4 \mathrm{M})$ & $\mathrm{U}(\$ 0.4 \mathrm{M}, \$ 0.5 \mathrm{M})$ & $\mathrm{U}(\$ 0.5 \mathrm{M}-\$ 0.7 \mathrm{M})$ \\
\hline$f c_{k, u}^{K}$ & $\mathrm{U}(\$ 0.8 \mathrm{M}-\$ 1.5 \mathrm{M})$ & $\mathrm{U}(\$ 1.5 \mathrm{M}-\$ 2.5 \mathrm{M})$ & $\mathrm{U}(\$ 2.5 \mathrm{M}-\$ 4 \mathrm{M})$ \\
\hline $\operatorname{cap}_{u}^{F}$ & $\mathrm{U}(200 \mathrm{~K}-400 \mathrm{~K})$ & U (400K-600K) & $\mathrm{U}(600 \mathrm{~K}-1000 \mathrm{~K})$ \\
\hline $\operatorname{cap}_{u}^{D}$ & $\mathrm{U}(100 \mathrm{~K}-200 \mathrm{~K})$ & U (200K-300K) & $\mathrm{U}(300 \mathrm{~K}-400 \mathrm{~K})$ \\
\hline $\operatorname{cap}_{u}^{I}$ & U (200K-400K) & $\mathrm{U}(400 \mathrm{~K}-600 \mathrm{~K})$ & $\mathrm{U}(600 \mathrm{~K}-800 \mathrm{~K})$ \\
\hline $\operatorname{cap}_{u}^{K}$ & $\mathrm{U}(100 \mathrm{~K}-400 \mathrm{~K})$ & U (400K-600K) & $\mathrm{U}(600 \mathrm{~K}-800 \mathrm{~K})$ \\
\hline $\operatorname{cap}_{u}^{J}$ & $\mathrm{U}(100 \mathrm{~K}-400 \mathrm{~K})$ & U (400K-600K) & $\mathrm{U}(600 \mathrm{~K}-800 \mathrm{~K})$ \\
\hline$L B R_{f, u}^{F}$ & $\mathrm{U}(100-150)$ & $\mathrm{U}(200-300)$ & $\mathrm{U}(300-500)$ \\
\hline$L B R_{d, u}^{D}$ & $\mathrm{U}(30-60)$ & $\mathrm{U}(60-100)$ & U (100-150) \\
\hline$L B R_{k, u}^{K^{u}}$ & $\mathrm{U}(30-50)$ & $\mathrm{U}(50-70)$ & $\mathrm{U}(70-100)$ \\
\hline$L B R_{i, u}^{I, n}$ & $\mathrm{U}(10-30)$ & $\mathrm{U}(30-50)$ & $\mathrm{U}(50-70)$ \\
\hline$L B R_{j, u}^{J}$ & $\mathrm{U}(10-30)$ & $\mathrm{U}(30-50)$ & $\mathrm{U}(50-70)$ \\
\hline
\end{tabular}

Table 6. Other parameters.

\begin{tabular}{cc}
\hline$r_{m, p}^{F}$ & $\mathrm{~m} 1, \mathrm{U}(4-6) ; \mathrm{m} 2, \mathrm{U}(14-17) ; \mathrm{m3},(4-6) ; \mathrm{m} 4, \mathrm{U}$ \\
$f_{c}^{S}$ & $(9-12) ; \mathrm{m} 5, \mathrm{U}(10-12)$ \\
$c c_{p, c}^{S}$ & $\mathrm{U}(\$ 1000-\$ 1200)$ \\
$r t c 1_{p, k}^{k}$ & $\mathrm{U}(\$ 30-\$ 50)$ \\
$r t c 2_{p, k}^{k}$ & $\mathrm{U}(\$ 40-\$ 50)$ \\
$r c_{p, j}^{J}$ & $\mathrm{U}(\$ 45-\$ 55)$ \\
$i c_{p, w}^{W}$ & $\mathrm{U}(\$ 5-\$ 10)$ \\
$e_{f}^{F}$ & $\mathrm{U}(\$ 3-\$ 8)$ \\
$e_{w}^{W}$ & $\mathrm{U}(9-10) \mathrm{kg}$ \\
$e_{k}^{K}$ & $\mathrm{U}(10-12) \mathrm{kg}$ \\
$e_{j}^{J}$ & $\mathrm{U}(6-7) \mathrm{kg}(1-2) \mathrm{kg}$ \\
$\rho_{p}^{0}$ & $\mathrm{U}(\$ 450-\$ 550)$ \\
$\rho_{p}^{1}$ & $\mathrm{U}(\$ 200-\$ 300)$ \\
$\rho_{p}^{2}$ & $\mathrm{U}(\$ 150-\$ 200)$ \\
$\rho_{p}^{r}$ & $\mathrm{U}(\$ 25-\$ 30)$ \\
\hline
\end{tabular}

\subsection{Computational Results and Sensitivity Analysis}

In order to evaluate the performance of the proposed model, first, the bi-objective version of the model is solved by considering the maximization of the profit and minimization of the environmental impact for different values of $\lambda$. The model is solved using GAMS software and a PC with the following configuration: Intel ${ }^{\circledR}$ Core $^{\mathrm{TM}}$ i7-1165G7 Processor $(2.80 \mathrm{GHz})$ and $16 \mathrm{~GB}$ DDR4 3200MHz.

Figures 5 and 6 illustrate the changes in objective functions with respect to changes to the risk factor $(\lambda)$. It can be observed that by increasing $\lambda$, meaning, by penalizing the deviation of the objective function from the mean value (reducing the risk taken by DM), both total profit and scenario variability are decreased. The optimality robustness of the model can be controlled by adjusting the risk factor $(\lambda)$ to DM's preferences. Reducing the risk factor to the lowest $(\lambda=0)$ results in the highest profit and highest variability. 


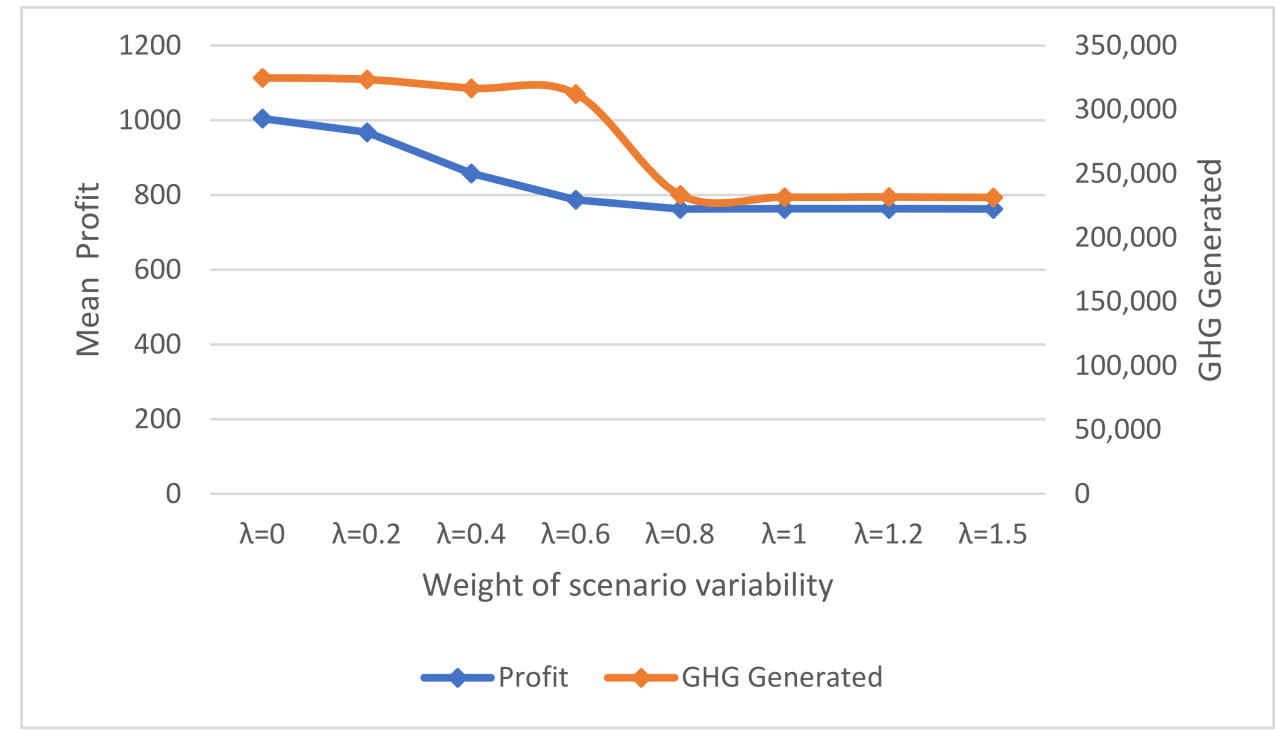

Figure 5. Value of objective functions for different value of $\lambda$.

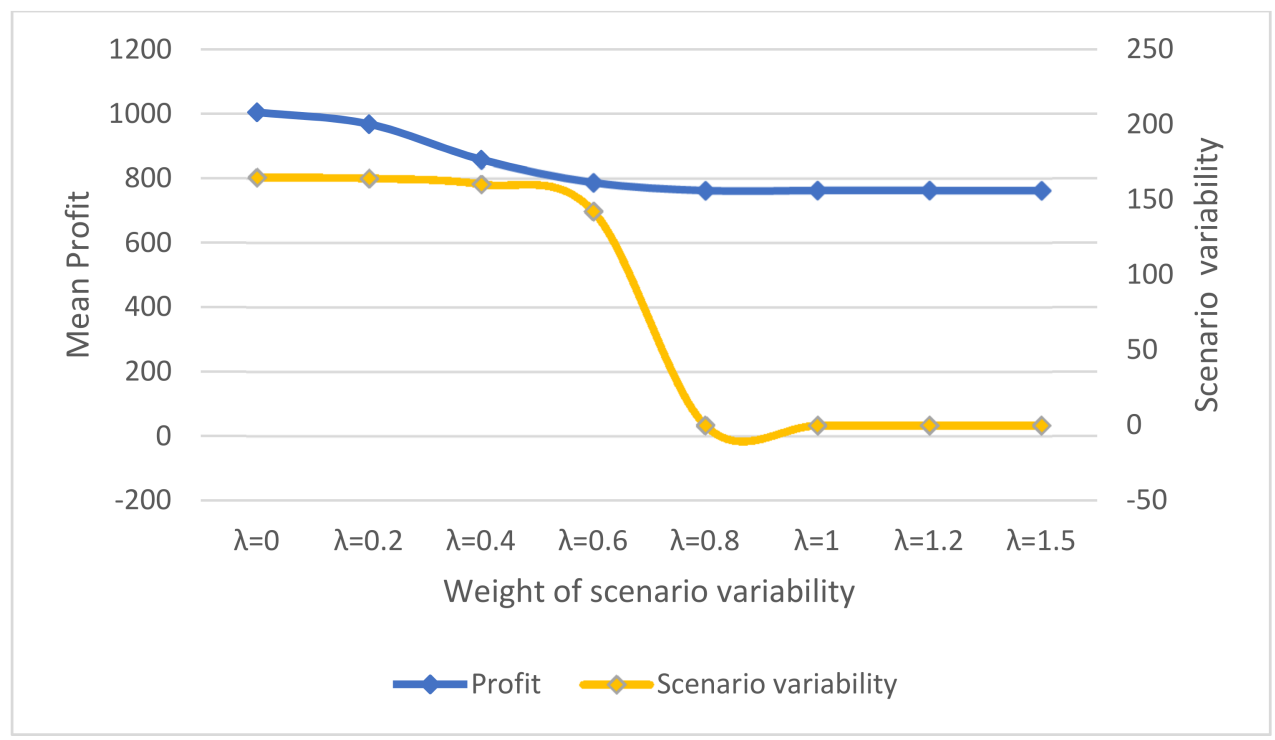

Figure 6. Mean value and scenario variability for different value of $\lambda$.

Table 7 shows the optimum configuration of the network, using the mean approach $(\lambda=0)$. In this configuration, two additional production centers will be open at high capacity and five additional distribution centers will be open, namely two at low, two at medium, and one at high capacity, to cope with the demand. In the reverse logistics, six collection centers will be open at low capacity; three retreading centers will be open, one at medium and two at high capacity; and two recycling centers open at low capacity. The network flow for this configuration is illustrated in Figure 7. The penalty cost for unsatisfied demand or shortage $(\omega)$ is another tool at DM's disposal to manage shortage in the system. An increase in the value of $\omega$ results in a decrease in mean profit and an increase in feasibility robustness. It can be seen in the optimum configuration of the network that the appropriate capacity levels of the open centers were determined in order to eliminate the shortage. The optimum confidence level of the fuzzy constraints $(\mathbb{P})$ was also determined based on the objective functions and the constraints which resulted in zero violation of fuzzy constraints $(\vartheta(\mathbb{P}))$. 


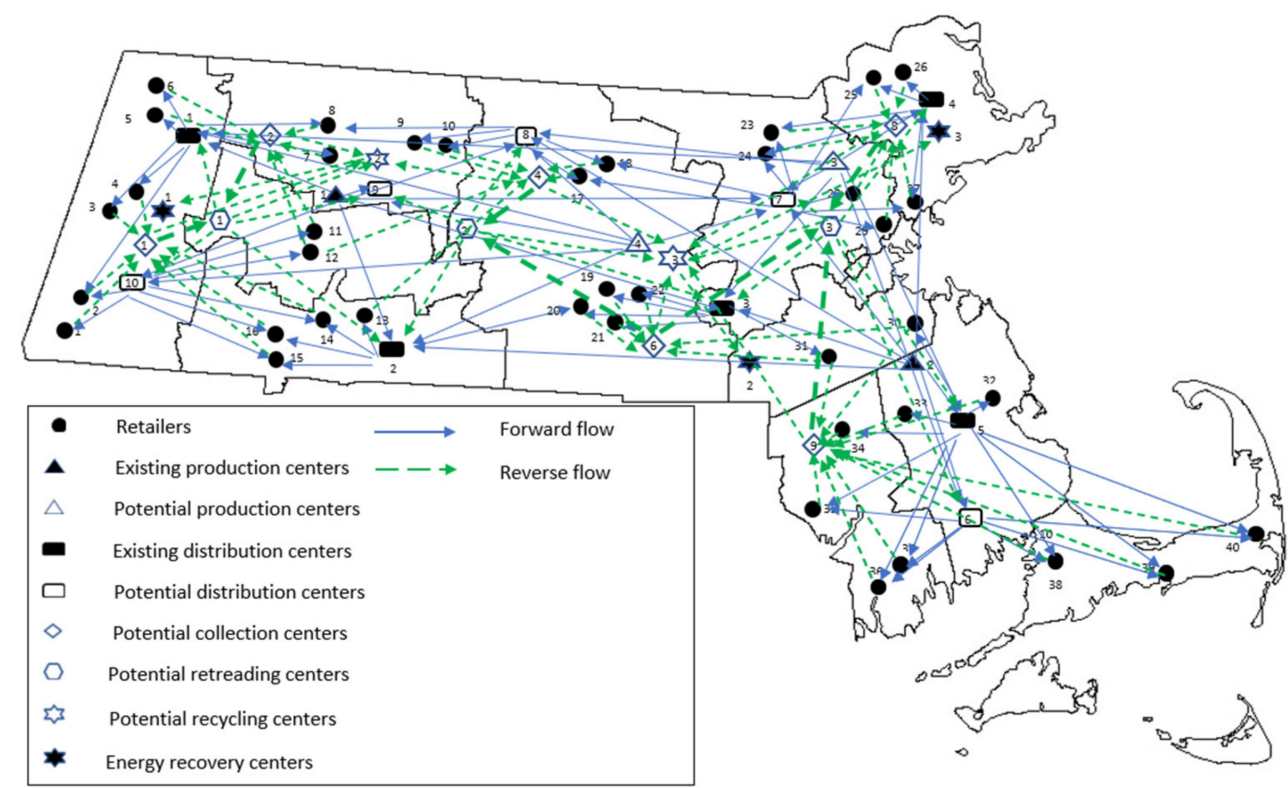

Figure 7. Optimum network configuration and product flow.

Table 7. Summary of optimum results, using mean approach.

\begin{tabular}{cc}
\hline Value of Coefficients & $\lambda=0, \omega=0, \Psi=0$ \\
\hline Objective 1 (profit, million dollar) & 1004 \\
Objective 2 (total GHG $=\mathrm{kg}$ ) & 324,893 \\
Objective 3 (total job opportunities) & 1600 \\
Production Centers & Production Centers 3 and 4 open at high capacity \\
Distribution Centers & Distribution Centers 6 and 7 open at low capacity \\
& Distribution Centers 8 and 9 open at medium ca-pacity \\
Collection Centers & Distribution Center 10 open at high capacity \\
Retreading Centers & Collection Centers $1,2,4,6,8$, and 9 open at low capacity \\
Recycling Centers & Retreading Center 2 open at medium capacity \\
Suppliers being utilized & Retreading Centers 1 and 3 open at high capacity \\
$\theta_{\xi}($ scenario variability) & Recycling Centers 2 and 3 open at low capacity \\
$\Delta_{\xi}$ (shortage) & Suppliers $1,3,4$, and 5 \\
$\vartheta(\mathbb{P})$ & 164.926 \\
$\mathbb{P}($ confidence level of fuzzy constraints) & 0 \\
& 0 \\
& $\mathbb{P}_{1}=1 / \mathbb{P}_{2}=0.5 / \mathbb{P}_{3}=0.5 / \mathbb{P}_{4}=1 / \mathbb{P}_{5}=1 / \mathbb{P}_{6}=1$ \\
\hline
\end{tabular}

Further sensitivity analysis is performed to evaluate the impact of changes to the problem parameters on the optimal solution and the model behavior. In that respect, we evaluate the impact of increasing the demand by $20 \%$ without making any changes to the number of potential facilities. As illustrated in Table 8, an increase in demand has resulted in an increase in total profit and GHG production which is the result of an increase in production. The capacity of one of the distribution centers has also increased from low to high level. It can be noted that there is some unsatisfied demand due to the capacity constraint at production centers.

We further evaluate the model by increasing the demand by $20 \%$ and also considering opening an additional production facility in order to eliminate the bottle neck at production centers. As it is shown in Table 9, the total profit further increased, the new production facility is open at a high-capacity level, and the capacity of the other two production facilities decreased to medium, in order to adjust with the overall demand, which resulted in zero unsatisfied demand. There was also an increase in the capacity of distribution centers, collection centers, recycling centers, and retreading centers. This is reflected in the total number of the job opportunities created along the network. 
Table 8. Summary of optimum result (demand increased by $20 \%$ ).

\begin{tabular}{|c|c|}
\hline Value of Coefficients & $\lambda=0, \omega=0, \Psi=0$ \\
\hline Objective 1 (profit, million dollar) & 1115 \\
\hline Objective 2 (total $\mathrm{GHG}=\mathrm{kg}$ ) & 348,253 \\
\hline Objective 3 (total job opportunities) & 1715 \\
\hline Production Centers & Production Centers 3 and 4 open at high capacity \\
\hline Distribution Centers & $\begin{array}{l}\text { Distribution Centers } 6 \text { open at low capacityDistribution Centers } \\
8 \text { and } 9 \text { open at medium capacityDistribution Centers } 7 \text { and10 } \\
\text { open at high capacity }\end{array}$ \\
\hline Collection Centers & Collection Centers $1,2,4,6,8$, and 9 open at low capacity \\
\hline Retreading Centers & $\begin{array}{l}\text { Retreading Center } 2 \text { open at medium capacityRetreading } \\
\text { Centers } 1 \text { and } 3 \text { open at high capacity }\end{array}$ \\
\hline Recycling Centers & Recycling Centers 2 and 3 open at low capacity \\
\hline Suppliers being utilized & Suppliers $1,3,4$, and 5 \\
\hline$\theta_{\tilde{\zeta}}$ (scenario variability) & 184.695 \\
\hline$\Delta_{\xi}$ (shortage) & 107,151 \\
\hline$\vartheta(\mathbb{P})$ & 0 \\
\hline $\mathbb{P}$ (confidence level of fuzzy constraints) & $\mathbb{P}_{1}=1 / \mathbb{P}_{2}=1 / \mathbb{P}_{3}=1 / \mathbb{P}_{4}=1 / \mathbb{P}_{5}=1 / \mathbb{P}_{6}=1$ \\
\hline
\end{tabular}

Table 9. Summary of optimum result (demand increase by $20 \%$ and considering opening an additional production center).

\begin{tabular}{|c|c|}
\hline Value of Coefficients & $\lambda=0, \omega=0, \Psi=0$ \\
\hline Objective 1 (profit, million dollar) & 1160 \\
\hline Objective 2 (total GHG, kg) & 358,321 \\
\hline Objective 3 (total job opportunities) & 1980 \\
\hline Production Centers & $\begin{array}{c}\text { Production Centers } 3 \text { and } 4 \text { open at medium capacityProduction } \\
\text { Centers } 5 \text { open at high capacity }\end{array}$ \\
\hline Distribution Centers & Distribution Centers $6,7,8,9$, and 10 open at high capacity \\
\hline Collection Centers & $\begin{array}{l}\text { Collection Centers } 1,2,4,8 \text {, and } 9 \text { open at low } \\
\text { capacityCollection Center } 6 \text { open at medium capacity }\end{array}$ \\
\hline Retreading Centers & $\begin{array}{c}\text { Retreading Center } 2 \text { open at low capacityRetreading Center } 2 \\
\text { open at medium capacityRetreading Centers } 1 \text { and } 3 \text { open at } \\
\text { high capacity }\end{array}$ \\
\hline Recycling Centers & Recycling Centers $1,2,3$, and 5 open at low capacity \\
\hline Suppliers being utilized & Suppliers $1,2,3,4$, and 5 \\
\hline$\theta_{\tilde{\zeta}}$ (scenario variability) & 190.680 \\
\hline$\Delta_{\tau}$ (shortage) & 0 \\
\hline$\vartheta(\mathbb{P})$ & 0 \\
\hline $\mathbb{P}$ (confidence level of fuzzy constraints) & $\mathbb{P}_{1}=1 / \mathbb{P}_{2}=0.5 / \mathbb{P}_{3}=0.5 / \mathbb{P}_{4}=1 / \mathbb{P}_{5}=1 / \mathbb{P}_{6}=1$ \\
\hline
\end{tabular}

In this section, the model is evaluated by optimizing all three objective functions, which are (1) maximizing the total profit, (2) minimizing the environmental impacts, and (3) maximizing the total number of jobs created along the network. As illustrated in Table 10, the total number of job opportunities has been increased by opening more centers at a high-capacity level. This has resulted in a reduction in the total profit due to the opening cost of the new facilities. The total amount of GHG remains unchanged, as it is directly correlated with the demand and the quantity of products being processed in each center. The overall unsatisfied demand remains at zero. The optimum confidence level of fuzzy constraints is obtained, which results in zero violation of fuzzy constraints $(\vartheta(\mathbb{P})$ ). 
Table 10. Summary of optimum result (maximizing the social benefits).

\begin{tabular}{|c|c|}
\hline Value of Coefficients & $\lambda=0, \omega=0, \Psi=0$ \\
\hline Objective 1 (profit, million dollar) & 998 \\
\hline Objective 2 (total GHG =kg) & 320,563 \\
\hline Objective 3 (total job opportunities) & 2452 \\
\hline Production Centers & Production Centers 3 and 4 open at high capacity \\
\hline Distribution Centers & Distribution Centers $6,7,8,9$, and 10 open at high capacity \\
\hline Collection Centers & Collection Centers 1, 2, 4, 6, 8, and 9 open at high capacity \\
\hline Retreading Centers & Retreading Centers $1,2,3$, and 4 open at high capacity \\
\hline Recycling Centers & Recycling Centers $1,2,3,4$, and 5 open at high capacity \\
\hline Suppliers being utilized & Suppliers $1,3,4$, and 5 \\
\hline$\theta_{\xi}$ (scenario variability) & 168.635 \\
\hline$\Delta_{\xi}($ shortage $)$ & 0 \\
\hline$\vartheta(\mathbb{P})$ & 0 \\
\hline $\mathbb{P}$ (confidence level of fuzzy constraints) & $\mathbb{P}_{1}=1 / \mathbb{P}_{2}=1 / \mathbb{P}_{3}=1 / \mathbb{P}_{4}=0.5 / \mathbb{P}_{5}=0.5 / \mathbb{P}_{6}=0.5$ \\
\hline
\end{tabular}

\subsection{The Managerial Implication}

There are many decision factors facing the DMs in designing a complex closed-loop supply-chain network. Consideration of disruption risk in addition to operational risk is one of the biggest challenges facing the DMs and the managers in supply-chain network. An example of disruption risk could be changes to economic condition, introduction of new technologies, changes in customer habit and preferences. Operational risks can be related to uncertainty in demand, procurement, production and transportation cost. RFSP is a powerful tool that provides flexibility to the DM to control these risks in the design of CLSC network simultaneously. By adjusting the risk factors $(\lambda)$ to the DM's preferences and their degree of conservatism, the optimality robustness of the network can be controlled. Feasibility robustness and the acceptable degree of violation of the constraints of the network can also be controlled by adjusting the value of $\Psi$ and $\omega$.

It is evident that this robust optimization method is capable of controlling scenario variability, possibilistic variability, and unsatisfied demand by adjusting the weighting scale based on the DM's preferences.

\section{Conclusions}

Recovery of EOL tires has been a challenge due to the large volume being produced and the material composition of the tires that makes them difficult to decompose. With all recent advancements in recycling options for EOL tires, a significant volume of them still ends up in the landfill. This indicates the importance of a need for a systemic, sustainable CLSC network for the tire industry. In this study, we developed a mixed-integer linear programming model for a CLSC network for the tire industry by considering several recovery options and taking into account the hybrid uncertainty associated with some parameters in the network, with the aim of optimizing the total profit, while minimizing the environmental impact and maximizing the social responsibility of the network. A robust fuzzy stochastic programming method was proposed to cope with the scenario-based uncertainty associated with the demand and fuzzy-based uncertainty associated with return rate, retreading rate, recycling rate, procurement cost, and production cost simultaneously.

To illustrate the performance and practicality of the proposed model, this model was applied for the design and development of a tire CLSC network in the Northeast USA. The result indicates the efficiency and applicability of the model in real-world problems and the feasibility of designing a profitable and environmentally friendly CLSC network for the tire industry. This model and proposed solution can be further extended and applied to the supply-chain network design of other industries.

One of the limitations of exact solution methods is their inability to solve large-scale problems; therefore, for a future study, a larger-scale problem using metaheuristics methods, with a focus on the impact of low-quality, low-cost imported tires and governmental inter- 
vention, regulations, and incentives on the performance and profitability of the network, can be considered.

Author Contributions: Investigation, M.T.; Methodology, M.T.; Supervision, S.M.G.; Validation, M.T.; Writing—original draft, M.T.; Writing—review \& editing, S.M.G. All authors have read and agreed to the published version of the manuscript.

Funding: This research received no external funding.

Acknowledgments: The authors would like to thank the reviewers for the insightful comments and feedback to enhance the quality of this article.

Conflicts of Interest: The authors declare no conflict of interest.

\section{References}

1. De Brito, M.P.; Dekker, R. A Framework for Reverse Logistics. In Reverse Logistics; Springer: Berlin, Heidelberg, $2004 ;$ pp. 3-27.

2. Daugherty, P.J.; Autry, C.W.; Ellinger, A.E. Reverse logistics: The relationship between resource commitment and program performance. J. Bus. Logist. 2001, 22, 107-123. [CrossRef]

3. Linton, J.; Klassen, R.; Jayaraman, V. Sustainable supply chains: An introduction. J. Oper. Manag. 2007, 25, 1075-1082. [CrossRef]

4. Selbes, M.; Yilmaz, O.; Khan, A.A.; Karanfil, T. Leaching of DOC, D.N., and inorganic constituents from scrap tires. Chemosphere 2015, 139, 617-623. [CrossRef] [PubMed]

5. Lin, C.; Huang, C.L.; Shern, C.-C. Recycling waste tire powder for the recovery of oil spills. Resour. Conserv. Recycl. 2008, 52, 1162-1166. [CrossRef]

6. Farrokh, M.; Azar, A.; Jandaghi, G.; Ahmadi, E. A novel robust fuzzy stochastic programming for closed loop supply chain network design under hybrid uncertainty. Fuzzy Sets Syst. 2018, 341, 69-91. [CrossRef]

7. Yu, C.S.; Li, H.L. A robust optimization model for stochastic logistic problems. Int. J. Prod. Econ. 2000, 64, 385-397. [CrossRef]

8. Pishvaee, M.S.; Torabi, S.A. A possibilistic programming approach for closed loop supply chain network design under uncertainty. Fuzzy Sets Syst. 2010, 161, 2668-2683. [CrossRef]

9. Ilgin, M.A.; Gupta, S.M. Environmentally conscious manufacturing and product recovery (ECMPRO): A review of the state of the art. J. Environ. Manag. 2010, 91, 563-591. [CrossRef]

10. Govindan, K.; Jafarian, A.; Khodaverdi, R.; Devika, K. Two echelon multiple vehicle location-routing problem with time windows for optimization of sustainable supply chain network of perishable food. Int. J. Prod. Econ. 2014, 15, 9-28. [CrossRef]

11. Devika, K.; Jafarian, A.; Nourbakhsh, V. Designing a sustainable closed loop supply chain network based on bottom line approach: A comparative metaheuristic hybridization technique. Eur. J. Oper. Res. 2014, 253, 594-615. [CrossRef]

12. Jindal, A.; Sangwan, K.S. Multi-objective fuzzy mathematical modelling of closed-loop supply chain considering economical and environmental factors. Ann. Oper. Res. 2017, 257, 95-120. [CrossRef]

13. Sarkar, B.; Tayyab, M.; Kim, N.; Habib, M.S. Optimal production delivery policies for supplier and manufacturer in a constrained closed-loop supply chain for returnable transport packaging through metaheuristic approach. Comput. Ind. Eng. 2019, 135, 987-1003. [CrossRef]

14. Sarkar, B.; Ullah, M.; Kim, N. Environmental and economic assessment of closed-loop supply chain with remanufacturing and returnable transport items. Comput. Ind. Eng. 2017, 111, 148-163. [CrossRef]

15. Dulman, M.; Gupta, S.M. Evaluation of Maintenance and EOL Operation Performance of Sensor-Embedded Laptops. Logistics 2018, 1, 3. [CrossRef]

16. Zhou, L.; Gupta, S.M. A Pricing and Acquisition Strategy for New and Remanufactured High-Technology Products. Logistics 2019, 3, 8. [CrossRef]

17. Zhou, L.; Gupta, S.M. Value depreciation factors for new and remanufactured high-technology products: A case study on iPhones and iPads. Int. J. Prod. Res. 2020, 58, 7218-7249. [CrossRef]

18. Aldoukhi, M.; Gupta, S. A robust closed loop supply chain network design under different carbon emission policies. Pamukkale University. J. Eng. Sci. 2019, 25, 1020-1032.

19. Aldoukhi, M.; Gupta, S.M. Use of Maximal Covering Location Problem to Design a Closed Loop Supply Chain Network Under Product Substitution. Appl. Manag. Sci. 2020, 20, 71-96.

20. Fadhel, A.W.; Gupta, S.M. Carbon Emissions and Energy Balance in the Design of a Sustainable Food Waste Network. Int. J. Ind. Eng. Oper. Manag. 2020, 2, 63-77.

21. Gupta, E.V.; Mogale, D.G.; Tiwari, M.K. Optimal Control of Production and Maintenance Operations in Smart Custom Manufacturing Systems with Multiple Machines. IFAC PapersOnLine 2019, 52, 241-246. [CrossRef]

22. De, M.; Giri, B.C. Modeling a closed-loop supply chain with a heterogeneous fleet under carbon emission reduction policy. Transp. Res. Part E Logist. Transp. Rev. 2020, 133, 101813. [CrossRef]

23. Mogale, D.G.; Lahoti, G.; Jha, S.B.; Shukla, M.; Kamath, N.; Tiwari, M.K. Dual Market Facility Network Design under Bounded Rationality. Algorithms 2018, 11, 54. [CrossRef] 
24. Oliveira, L.S.; Machado, R.L. Application of optimization methods in the closed-loop supply chain: A literature review. J. Comb. Optim. 2021, 41, 357-400. [CrossRef]

25. Zahedi, A.; Salehi-Amiri, A.; Hajiaghaei-Keshteli, M.; Diabat, A. Designing a closed-loop supply chain network considering multi-task sales agencies and multi-mode transportation. Soft Comput. 2021, 1-33.

26. Guarnieri, P.; Camara, E.; Silva, L.; Vieira, B.O. How to Assess Reverse Logistics of e-Waste Considering a Multicriteria Perspective: A Model Proposition. Logistics 2020, 4, 25. [CrossRef]

27. Vieira, B.O.; Guarnieri, P.; Nofal, R.; Nofal, B. Multi-Criteria Methods Applied in the Studies of Barriers Identified in the Implementation of Reverse Logistics of E-Waste: A Research Agenda. Logistics 2020, 4, 11. [CrossRef]

28. Mogale, D.G.; Kumar, S.K.; Tiwari, M.K. Green food supply chain design considering risk and post-harvest losses: A case study. Ann. Oper. Res. 2020, 295, 257-284. [CrossRef]

29. Mogale, D.G.; Cheikhrouhou, N.; Tiwari, M.K. Modelling of sustainable food grain supply chain distribution system: A bi-objective approach. Int. J. Prod. Res 2019. [CrossRef]

30. Ullah, M.; Asghar, I.; Zahid, M.; Omair, M.; AlArjani, A.; Sarkar, B. Ramification of remanufacturing in a sustainable three-echelon closed-loop supply chain management for returnable products. J. Clean. Prod. 2021, 290, 125609. [CrossRef]

31. Ghadge, A.; Kara, M.; Mogale, D.G.; Choudhary, S.; Dani, S. Sustainability implementation challenges in food supply chains: A case of U.K. artisan cheese producers. Prod. Plan. Control. 2020. [CrossRef]

32. Gupta, S.M.; Güngör, A.; Govindan, K.; Özceylan, E.; Can Berk, K.; Piplani, R. Responsible \& sustainable manufacturing. Int. J. Prod. Res. 2020, 58, 7181-7182.

33. Sazvar, Z.; Zokaee, M.; Tavakkoli-Moghaddam, R.; Al-sadat Salari, S.; Nayeri, S. Designing a sustainable closed-loop pharmaceutical supply chain in a competitive market considering demand uncertainty, manufacturer's brand and waste management. Ann. Oper. Res. 2021, 1-32. [CrossRef]

34. Meade, L.; Sarkis, J. A conceptual model for selecting and evaluating third-party reverse logistics providers. Supply Chain Manag. Int. J. 2002, 7, 283-295. [CrossRef]

35. Ahmadi, A.; Amin, S. An integrated chance-constrained stochastic model for a mobile phone closed-loop supply chain network with supplier selection. J. Clean. Prod. 2019, 226, 988-1003. [CrossRef]

36. Fazli-Khlaf, M.; Chaharsoohi, S.; Pishvaee, S. A new robust possibilistic programming model for reliable supply chain network design: A case study of lead- acid battery supply chain. RAIRO Oper. Res. 2019, 53, 1489-1512. [CrossRef]

37. Sabouhi, F.; Pishvaee, M.S.; Jabalameli, M.S. Resilient Supply Chain Design under Operational and Disruption Risks Considering Quantity Discount: A Case Study of Pharmaceutical Supply Chain. Comput. Ind. Eng. 2018, 126, 657-672. [CrossRef]

38. Mula, J.; Poler, R.; Garcia, J.P. MRP with flexible constraints: A fuzzy mathematical programming approach. Fuzzy Sets Syst. 2006, 157, 74-97. [CrossRef]

39. Mula, J.; Poler, R.; Garcia-Sabater, J.P. Material Requirement Planning with fuzzy constraints and fuzzy coefficients. Fuzzy Sets Syst. 2007, 158, 783-793. [CrossRef]

40. Pishvaee, M.S.; Razmi, J. Environmental supply chain network design using multi-objective fuzzy mathematical programming. Appl. Math. Model. 2012, 36, 3433-3446. [CrossRef]

41. Pishvaee, M.S.; Torabi, S.A.; Razmi, J. Credibility-based fuzzy mathematical programming model for green logistics design under uncertainty. Comput. Ind. Eng. 2012, 62, 624-632. [CrossRef]

42. Mulvey, J.M.; Vanderbei, R.J.; Zenios, S.A. Robust optimization of large-scale systems. Oper. Res. 1995, 43, 264-328. [CrossRef]

43. Pishvaee, M.S.; Razmi, J.; Torabi, S.A. Robust possibilistic programming for socially responsible supply chain network design: A new approach. Fuzzy Sets Syst. 2012, 206, 1-20. [CrossRef]

44. Mousazadeh, M.; Torabi, S.A.; Pishvaee, M.S.; Abolhassani, F. Health service network design: A robust possibilistic approach. Int Trans. Oper. Res. 2018, 25, 337-373. [CrossRef]

45. Tozanli, O.; Duman, G.M.; Kongar, E.; Gupta, S.M. Environmentally Concerned Logistics Operations in Fuzzy Environment: A Literature Survey. Logistics 2017, 1, 4. [CrossRef]

46. Vahdat, V.; Vahdatzad, M.A. Accelerated Benders' Decomposition for Integrated Forward/Reverse Logistics Network Design under Uncertainty. Logistics 2017, 1, 11. [CrossRef]

47. Prakash, S.; Kumar, S.; Soni, G.; Jain, V.; Rathore, A. Closed-loop supply chain network design and modeling under risks and demand uncertainty: An integrated robust optimiza-tion approach. Ann. Oper. Res. 2020, 290, 837-864. [CrossRef]

48. Yolmeh, A.; Saif, U. Closed-loop supply chain network design integrated with assembly and disassembly line balancing under uncertainty: An enhanced decomposition approach. Int. J. Prod. Res. 2020, 1-18. [CrossRef]

49. Wang, W.; Zhang, Y.; Zhang, W.; Gao, G.; Zhang, H. Incentive mechanisms in a green supply chain under demand uncertainty. J. Clean. Prod. 2018, 279, 123636. [CrossRef]

50. Tordecilla, R.D.; Juan, A.A.; Montoya-Torres, J.R.; Quintero-Araujo, C.L.; Panadero, J. Simulation-optimization methods for designing and assessing resilient supply chain networks under uncertainty scenarios: A review. Simul. Model. Pract. Theory 2021, 106, 102166. [CrossRef]

51. Ferrer, G. The economics of tire remanufacturing. Resour. Conserv. Recycl. 1997, 19, 221-255. [CrossRef]

52. Sasikumar, P.; Kannan, G.; Noorul Haq, A. A multi-echelon reverse logistics network design for product recovery-A case of truck tire remanufacturing. Int. J. Adv. Manuf. Technol. 2010, 49, 1223-1234. [CrossRef]

53. Chopra, S.; Meindl, P. Supply Chain Management: Strategy, Planning and Operation; Pearson: Boston, MA, USA, 2013 ; Volume 232. 
54. Yadollahinia, M.; Teimoury, E.; Paydar, M. Tire forward and reverse supply chain design considering customer relationship management. Resour. Conserv. Recycl. 2018, 138, 215-228. [CrossRef]

55. Amin, S.H.; Zhang, G.; Akhtar, P. Effects of uncertainty on a tire closed-loop supply chain network. Exp. Syst. Appl. 2017, 73, 82-91. [CrossRef]

56. Subulan, K.; Tas, A.S.; Baykasoğlu, A. Designing an environmentally conscious tire closed-loop supply chain network with multiple recovery options using interactive fuzzy goal programming. Appl. Math. Model. 2015, 39, 2661-2702. [CrossRef]

57. Derakhshan, Z.; Ghaneian, M.; Mahvi, A.; Gea, O.C.; Faramarzian, M.; Dehghani, M.; Ferrante, M. A new recycling technique for the waste tires reuse. Environ. Res. 2017, 158, 462-469. [CrossRef] [PubMed]

58. O'Brien, B.O.; North, B. A Review of Waste Tyre Gasification. J. Environ. Chem. Eng. 2017, 5, 10. [CrossRef]

59. Fathollahi-Fard, A.M.; Hajiaghaei-Keshteli, M. Hybrid optimizers to solve a tri-level programming model for a tire closed-loop supply chain network design. Appl. Soft Comput. 2018, 70, 701-722. [CrossRef]

60. Pedram, A.; Yusoff, N.; Udoncy, O.; Mahat, A.; Pedram, P.; Babolola, A. Integrated forward and reverse supply chain: A tire case study. Waste Manag. 2017, 60, 460-470. [CrossRef]

61. Lokesh, K.S.; Jain, P.K.; Sharma, A.K. Tactical supply chain planning for Tyre remanufacturing considering carbon tax policy. Int. J. Adv. Manuf. Technol. 2018, 97, 1505-1528.

62. Lokesh, K.S.; Jain, P.K.; Sharma, A.K. A fuzzy goal programme with carbon tax for Brownfield tyre remanufacturing supply chain planning. J. Clean. Prod. 2018, 198, 737-753.

63. Sahebjamnia, N.; Fathollahi-Fard, A.; Hajiaghaei-Keshteli, M. Sustainable tire closed-loop supply chain network design: Hybrid metaheuristic algorithms for large-scale networks. J. Clean. Prod. 2018, 196, 273-296. [CrossRef]

64. Abdolazimi, O.; Esfandarani, M.; Salehi, M.; Shishebori, D. Robust design of a multi-objective closed-loop supply chain by integrating on-time delivery, cost, and environmental aspects, case study of a Tire Factory. J. Clean. Prod. 2020, 264, 121566. [CrossRef]

65. Mehrjerdi, Y.Z.; Shafiee, M. A Resilient and sustainable closed-loop supply chain using multiple sourcing and information sharing strategies. J. Clean. Prod. 2020, 289, 125141. [CrossRef]

66. Lokesh, K.S.; Jain, P.K.; Sharma, A.K. A fuzzy goal programme-based sustainable Greenfield supply network design for tyre retreading industry. Int. J. Adv. Manuf. Technol. 2020, 108, 2855-2880.

67. Gupta, V.K.; Nayak, A.; Agarwal, S.; Chaudhary, M.; Tyagi, I. Removal of Ni (II) ions from water using scrap tire. J. Mol. Liq. 2014, 190, 215-222. [CrossRef]

68. Derakhshan, Z.; Ehrampoush, M.H.; Faramarzian, M.; Ghaneian, M.T.; Mahvi, A.H. Waste tire chunks as a novel packing media in a fixed-bed sequence batch reactor: Volumetric removal modeling. Desalin. Water Treat. 2017, 64, 40-47. [CrossRef]

69. Battista, M.; Gobetti, A.; Agnelli, S.; Ramorino, G. Post-consumer tires as a valuable resource: Review of different types of material recovery. Environ. Technol. Rev. 2021, 10, 1-25. [CrossRef]

70. Conti, G.O.; Heibati, B.; Kloog, I.; Fiore, M.; Ferrante, M. A review of AirQ Models and their applications for forecasting the air pollution health outcomes. Environ. Sci. Pollut. Res. Int. 2017, 24, 6426-6445. [CrossRef]

71. Sciacca, S.; Conti, G.O. Mutagens and carcinogens in drinking water. Med. J. Nutr. 2009, 2, 157-162.

72. Dehghani, M.; Keshtgar, L.; Javaheri, M.R.; Derakhshan, Z.; Conti, G.O.; Zuccarello, P.; Ferrante, M. The effects of air pollutants on the mortality rate of lung cancer and leukemia. Mol. Med. 2017, 15, 3390-3397. [CrossRef]

73. Salehi, F.; Mahootchi, M.; Husseini, S.M.M. Developing a robust stochastic model for designing a blood supply chain network in a crisis: A possible earthquake in Tehran. Ann. Oper. Res. 2019, 283, 679-703. [CrossRef]

74. Kacprzyk, J.; Orlovski, S.A. Optimization Models Using Fuzzy Sets and Possibility Theory; Springer Science \& Business Media: Berlin, Germany, 2013; Volume 4.

75. $\mathrm{Xu}, \mathrm{J}$; Z Zhou, X. Approximation based fuzzy multi-objective models with expected objectives and chance constraints: Application to earth-rock work allocation. Inf. Sci. 2013, 238, 75-95. [CrossRef]

76. Zahiri, B.; Jula, P.; Tavakkoli-Moghaddam, R. Design of a pharmaceutical supply chain network under uncertainty considering perishability and substitutability of products. Inf. Sci. 2018, 423, 257-283. [CrossRef]

77. Savadkoohi, E.; Mousazadeh, M.; Torabi, S.A. A possibilistic location-inventory model for multi-period perishable pharmaceutical supply chain network design. Chem. Eng. Res. Des. 2018, 138, 490-505. [CrossRef]

78. Heydari, J.; Sabbaghnia, A.; Razmi, J. A dynamic bi-objective model for after disaster blood supply chain network design; a robust possibilistic programming ap-proach. J. Ind. Syst. Eng. 2018, 11, 16-28.

79. Sadghiani, N.S.; Torabi, S.; Sahebjamnia, N. Retail supply chain network design under operational and disruption risks. Transp. Res. Part E Logist. Transp. Rev. 2015, 75, 95-114. [CrossRef]

80. Zhang, P.; Zhang, W.-G. Multiperiod mean absolute deviation fuzzy portfolio selection model with risk control and cardinality constraints. Fuzzy Sets Syst. 2014, 255, 74-91. [CrossRef]

81. Mavrotas, G. Effective implementation of the $\mathcal{\varepsilon}$-constraint method in multi-objective mathematical programming problems. Appl. Math. Comput. 2009, 213, 455-465. [CrossRef]

82. Aghaei, J.; Amjady, N.; Shayanfar, H.A. Multi-objective electricity market clearing considering dynamic security by lexicographic optimization and augmented epsilon constraint method. Appl. Soft Comput. 2011, 11, 3846-3858. [CrossRef] 